\title{
Regularity-Guaranteed Transformation Estimation in Medical Image Registration
}

\author{
A thesis presented to \\ the faculty of \\ the Russ College of Engineering and Technology of Ohio University \\ In partial fulfillment \\ of the requirements for the degree \\ Master of Science
}

\section{Bibo Shi}

August 2011

(C) 2011 Bibo Shi. All Rights Reserved. 
This thesis titled

Regularity-Guaranteed Transformation Estimation in Medical Image Registration

by

BIBO SHI

has been approved for

the Department of Chemical and Biomolecular Engineering and the Russ College of Engineering and Technology by

Jundong Liu

Associate Professor of Electrical Engineering and Computer Science

Dennis Irwin

Dean of Russ College of Engineering and Technology 


\begin{abstract}
SHI, BIBO, M.S., August 2011, Biomedical Engineering

$\underline{\text { Regularity-Guaranteed Transformation Estimation in Medical Image Registration (64 pp.) }}$

Director of Thesis: Jundong Liu

In addition to seeking geometric correspondence between the inputs, a legitimate medical image registration algorithm should also keep the estimated transformation meaningful or regular. In this thesis, we present a mathematically sound formulation that explicitly controls the transformation to keep each grid in a meaningful shape over the entire geometric matching procedure. The deformation regularity conditions are enforced by maintaining all the moving neighbors as non-twist grids. In contrast to similar work, we differentiate and formulate the convex and concave folding cases under an efficient and straightforward point-to-line/surface orientation framework, and use equality constraints to guarantee grid regularity and prevent folding. The equality constrained optimization problem is efficiently solved using the augmented Lagrangian Mulplier method. Experiments on human brain MR images are presented to show the improvements made by our model over the popular Demon's and DCT-based registration algorithms. Extension to develop a clinical registration package including the regularity guaranteed conditions is also explored.
\end{abstract}

Approved:

Jundong Liu

Associate Professor of Electrical Engineering and Computer Science 


\section{ACKNOWLedgements}

During my Master study at Ohio University, I have been privileged to have Dr. Jundong Liu as my advisor. I am deeply grateful to Dr. Liu for his insightful advice, patience and all the discussion with me, which help me build a ground foundation for my future Ph.D study.

I also thank my committee members, Drs. Goetz, Marling, and Aizicovici, for their time and efforts to supervise my thesis. Their valuable comments have made my thesis better.

My gratitude is also with my labmates, Shuisheng Xie, Huihui Xu, and David Days, who always provide me with the inspiring disscussions. Special thanks also go to my best friends, who are in Athens and back in China. Thank you all for sharing my difficult and joyful memories with me, for always trusting me.

To my parents, my families, you are the reason that I want to be better. Thanks for your always unselfish love and support.

This work was supported by OU Biomedical Engineering Program, and has been published and presented in MIUA'11 and GLBIO'11. 


\section{TABle of Contents}

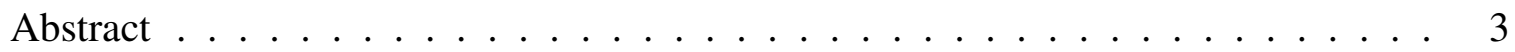

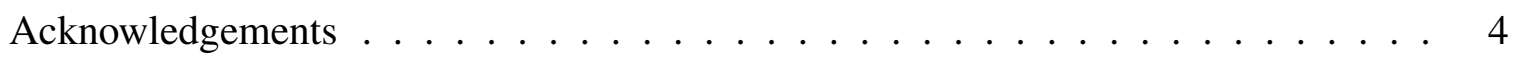

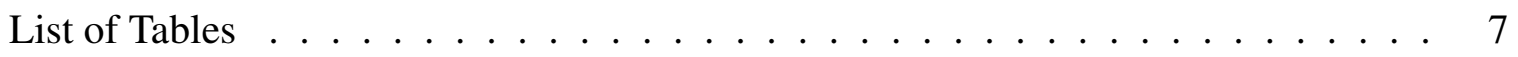

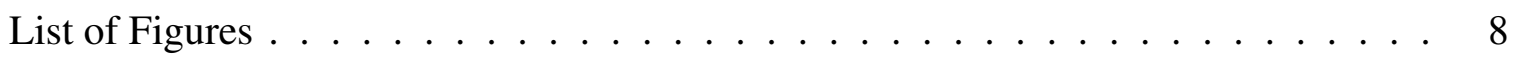

1 Introduction . . . . . . . . . . . . . . . . . . 9

1.1 Medical Image Registration $\ldots \ldots \ldots$

1.1.1 Registration Framework as an Optimization Problem . . . . . . . 11

1.1.2 Registration as an Ill-Posed Problem . . . . . . . . . . . . . . 14

1.2 Motivation for our Regularity-Guaranteed Image Registration . . . . . . . 16

1.3 Thesis Contribution . . . . . . . . . . . . . . . . . . 17

1.4 Thesis Outline . . . . . . . . . . . . . . . . . . . . . . 18

2 Related work . . . . . . . . . . . . . . . . . . . . . . . . 19

2.1 Regularization in Image Registration $\ldots \ldots \ldots$

2.1.1 Regularization on Smoothness . . . . . . . . . . . . . . . . . . 19

2.1.1.1 Basis Function Expansion based . . . . . . . . . 20

2.1.1.2 Physical Model based . . . . . . . . . . . . . . . 24

2.1.2 Regularization on Topology . . . . . . . . . . . . . . . . 27

2.2 Specific Requirements for Neurodegenerative Diseases . . . . . . . . . 30

3 Non-Twist Regularization Framework . . . . . . . . . . . . . . . . . 33

3.1 Non-Twist Regularization . . . . . . . . . . . . . . . . . . . . 33

3.2 Integration with Registration Framework . . . . . . . . . . . . . 35

3.3 Numerical Solutions for Constrained Optimization Problem . . . . . . . . . 37

3.3.1 Constrained Optimization _ . . . . . . . . . . . . . . 37

3.3.2 Practical Numerical Solutions . . . . . . . . . . . . . . . . . 39

4 Experimental Results . . . . . . . . . . . . . . . . . . . . . . . 44

4.1 Synthetic Sinusoidal Deformation . . . . . . . . . . . . . . . 44

4.2 Comparative Results: Demon's vs Demon's + Non-Twist . . . . . . . . . . 45

4.3 Comparative Results: DCT vs DCT + Non-Twist . . . . . . . . . . 51

4.4 Preliminary Design for GUI $\ldots \ldots \ldots \ldots \ldots \ldots$

5 Conclusion and Future Work . . . . . . . . . . . . . . . 55

References . . . . . . . . . . . . . . . . . . 57 
Appendix: Matlab Code . . . . . . . . . . . . . . . . . . 61 


\section{List OF TABLES}

2.1 The Toll of Selected Brain and Nervous System Disorder on Americans _ . . . 31

4.1 Sum of Differences: Demon's vs Demon's + Non-Twist . . . . . . . . . . . 49

4.2 Average Angle Error: Demon's vs Demon's + Non-Twist . . . . . . . . . . . . 51

4.3 Sum of Differences: DCT vs DCT + Non-Twist . . . . . . . . . . . . . 51

4.4 Average Angle Error: DCT vs DCT + Non-Twist . . . . . . . . . . . . 52 


\section{List Of Figures}

1.1 Multi-modal analysis: registration of MRI and PET images, from up to down: MRI, PET, and after fusion; from left to right: horizontal plane, coronal plane, and sagittal plane (from the webpage of Department of Clinical Neurosciences, University of Cambridge, available online at http://www.neuroscience.cam.ac.uk/). . . . . . . . 12

1.2 Components of image registration . . . . . . . . . . . . . . . . 13

1.3 Infinite solutions for image registration . . . . . . . . . . . . . 14

2.1 The lowest frequency basis functions of $2 \mathrm{D}$ DCT $\ldots \ldots \ldots$. . . . . . . . 23

2.2 Comparison of a normal aged brain (left) and an Alzheimer's patient's brain (right) (from the webpage of "Alzheimer's Disease Education and Referral Center, a service of the National Institute on Aging."). . . . . . . . . . . . . . . . . . . . . . 32

3.1 Illustration of possible grid update scenarios. (a) starting grid; (b) convex case; (c) concave case; $(\mathrm{d})$ twisting case, which should be avoided. . . . . . . . . . . . . 34

3.2 Point-line orientation. (a) Positive orientation: $P 0$ is on the right side of the line $P 2 P 1$;

(b) Negative orientation: $P 0$ is on the left side of the line $P 2 P 1 \ldots \ldots 34$

3.3 Algorithm of the quadratic penalty method . . . . . . . . . . . . . 41

3.4 Algorithm of the logrithmic barrier method . . . . . . . . . . . . . . . . . . . . . . . . . . . . . . .

3.5 Algorithm of the augmented Lagrangian method . . . . . . . . . . . . . . . . 42

4.1 MR brain image. (a) Reference image (b) Floating image (c) ground truth of the deformation field (d) Original differences . . . . . . . . . . . . . 46

4.2 Registration results by the Demon's alone. (The left figures are registered floating images, and the right figures are the corresponding transformation fields, with increasing smoothing- $\sigma.) \ldots \ldots$. . . . . . . . . 47

4.3 Registration results by the Demon's $(\sigma=6)$ with Non-Twist regularity. . . . . . 48

4.4 Differences between the reference image and registered image. . . . . . . . . 50

4.5 Vector Field between the reference image and registrated image. . . . . . . . . 50

4.6 Registration results by DCT alone and DCT with Non-Twist regularity. (The left figures are regitered floating images, and the right figures are the corresponding transformation fields. ) . . . . . . . . . . . 52

4.7 Differences between the reference image and registered image. . . . . . . . . . 53

4.8 Vector Field between the reference image and registrated image. . . . . . . . . 53

4.9 Medical Image Registration Toolbox. . . . . . . . . . . . . . . . . . . 54 


\section{INTRODUCTION}

Medical imaging was pioneered by Roentgen through the discovery of the X-ray. The first non-invasive radiography on part of a human body, Mrs. Roentgen's hand, was taken on December 22, 1895, along with tremendous uncertainty or even fears [37]. Since then, medical imaging techniques have been on a booming stage, and have proven to be a robust and vital tool that assists clinicians in many aspects, including clinical diagnosis, monitoring disease progression, treatment verification, and surgical planning. While image acquisition is focused on designing more modalities and speeding up the imaging procedure, image analysis, the process to extract the useful information from the acquired image data, has been playing a more and more important role in the entire application spectrum. At present, research in medical image analysis is mainly focused on developing computational models and algorithms to analyze and quantify medical data, thereby exploring and maximizing their utilization in clinical settings.

One of the characteristics of medical data sets is their volume. Take Magnetic Resonance Imaging (MRI) as an example. During one imaging session, multiple scans, with different imaging protocols, of the same patient will be obtained. The combined data size could easily be as large as 1-2 Gigabytes. With the vast amount of medical image data produced everyday, a problem that commonly arises is how this data can be compared and effectively utilized.

Usually, the data sets are taken at different times on different machines (for both single modality or multi-modality cases). Inevitably there will be some motion between them. Image registration, the process of matching two or more images spatially, therefore becomes the prerequisite step for the complementary information to be effectively combined and integrated.

Besides medical applications, image registration has also been widely used in various other areas [48], e.g., remote sensing (multispectral classification, environmental 
monitoring, change detection, weather forecasting, creating super-resolution images, integrating information into geographic information systems (GIS)), cartography (map updating), and computer vision (target localization, automatic quality control), etc. Registration in medical applications, however, has some unique aspects, which stem from the unique properties of $3 \mathrm{D}$ medical data [18]: the modalities of image information that are acquired, the fully three-dimensional image data, the non-rigid nature of object motion and deformation, and the statistical variation of both the underlying normal and abnormal ground truth.

The following sections illustrate in detail a variety of aspects of the medical image registration problem. We start with the mathematical definition of image registration, and will touch upon the regularity issue within the deformation estimation procedure.

\subsection{Medical Image Registration}

According to a well accepted definition by D.L.G. Hill [17], registration is the process of "determining a transformation that can relate the position of features in one image or coordinate space with the position of the corresponding feature in another image or coordinate space. Typically, this space deformation between two images or coordinate spaces results from the following three manners of image acquisition [48]:

1. Different viewpoints (multi-view analysis): images of the same scene are acquired from different viewpoints;

2. Different times (multi-temporal analysis/ longitudinal analysis): images of the same scene are acquired at different times, possibly under different conditions;

3. Different sensors (multi-modal analysis): images of the same scene are acquired by different sensors. 
The latter two types of analysis are usually of more practical use in the modern clinical setting. Regarding longitudinal analysis, time series of medical images are acquired for various reasons [31], such as monitoring of brain shrinkage (long interval), monitoring of tumor growth (medium internal), post-operative monitoring of recovering (short interval), or relocating the mesh of virtual reality with organs of the patients during operation (ultrashort interval). On the other hand, multi-modal analysis provides the medical practitioners with a comprehensive base that usually merges both anatomical and functional information. Currently, the conventional anatomical imaging modalities are Ultrasound (US), X-Ray, Computed Tomography (CT), and Magnetic Resonance Imaging (MRI), while functional MRI, Positron Emission Tomography (PET), and Single Photon Emission Computed Tomography (SPECT) are used to depict the functional activities.

Figure 1.1 shows the structure and function combined in an individual with the posterior cortical atrophy variant of Alzheimer's disease. The structure is given with MRI (top), the function with PET (middle) and the two modalities fused together (bottom).

\subsubsection{Registration Framework as an Optimization Problem}

Typically, the process of image registration is formulated as an optimization problem, as shown in Figure 1.2. The inputs to the registration are: a reference (or static) image $R(x)$, and a floating (or moving) image $F(x)$, which will be changed over the deformation estimation process. An objective function $J$ will be optimized to determine a transformation field $T$ (assigned to each point $x$ ) that minimizes the difference between $F(T(x))$ and $R(x)$, where $F(T(x))$ is the transformed floating image.

$$
J(T(x)):=D(R(x), F(T(x)))
$$

Most registration algorithms contain the following four crucial components [9]: 


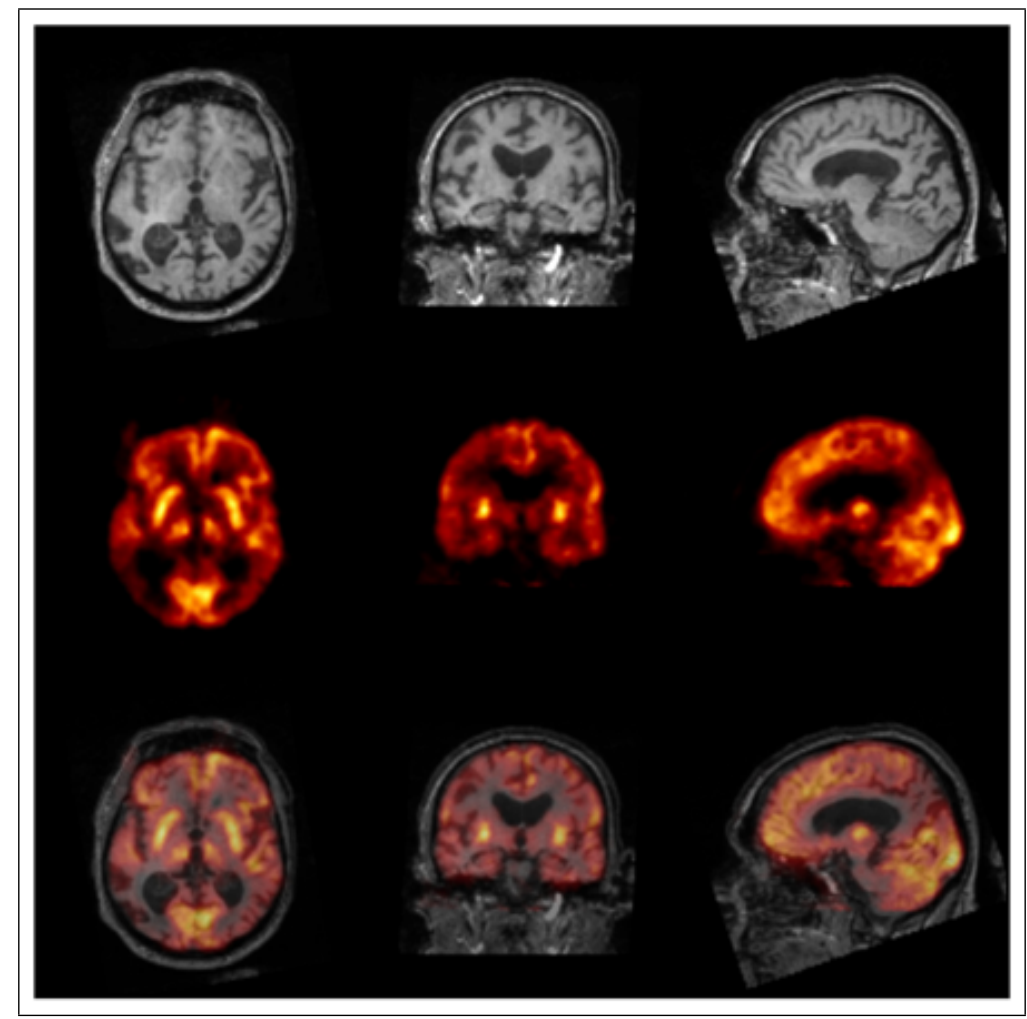

Figure 1.1: Multi-modal analysis: registration of MRI and PET images, from up to down: MRI, PET, and after fusion; from left to right: horizontal plane, coronal plane, and sagittal plane (from the webpage of Department of Clinical Neurosciences, University of Cambridge, available online at http://www.neuroscience.cam.ac.uk/).

1. Feature Space: The first step in registration is to decide what type of information extracted from the image would be used as the basis for matching. The common features used in registration are: raw intensity (most cases), edges, salient features (e.g., points of locally maxium curvature on contour lines, line intersections), statistical features (e.g., moment invariants), higher-level features, etc. In this thesis, we are mainly focused on intensity-based registration.

2. Similarity Metrics (term $D$ ): The second step for designing a registration method is the selection of a similarity metric, which is closely related to the chosen feature space. Similarity metrics are of significance for quantifying the similarity (or 


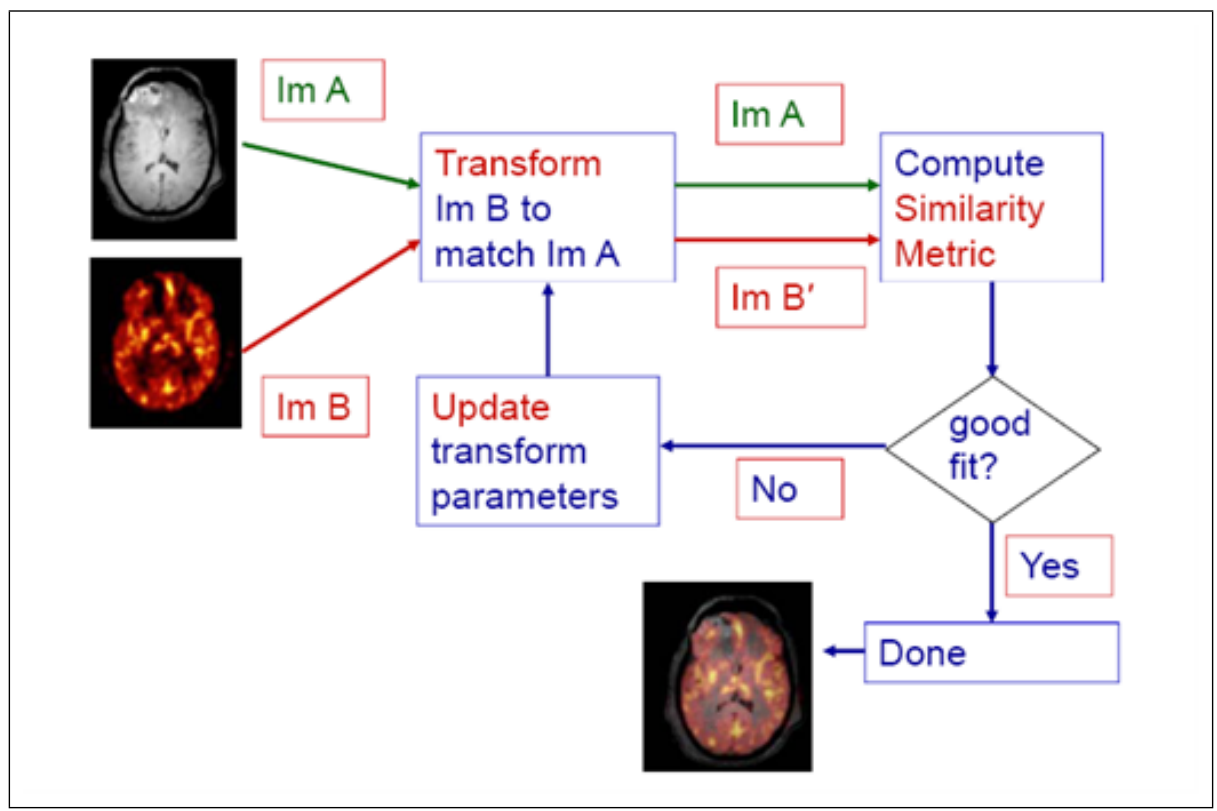

Figure 1.2: Components of image registration

difference) of two input images, measuring how well they are matched together. Sum of Squared Differences (SSD), Correlation Coefficient (CC), and Mutual Information (MI) are among the widely used metrics for medical image registration. The selection among them is highly dependent on the specific problem.

3. Transformation (term $T$ ): The transformation that needs to be determined through the optimization is the goal and outcome of the registration solution, which spatially maps the pixels/voxels of the floating and reference image. Usually, different models of transformation indicate different assumptions about the deformation and other variations presented in the images. Models can be grouped under different perspectives: global vs local, linear vs non-linear, or parametric vs non-parametric transformation. Details of two exemplary transformation models used in this thesis will be given in chapter 2 . 
4. Optimization/numerical strategy: Due to the large computational costs stemming from the volume of the medical images and/or the chosen dense transformation model, the numerical strategy to solve this optimization problem plays a determining role for both the efficiency and viability of the whole procedure. Starting with an initial set of parameters for the transformation field, the numerical strategy would iteratively search for the optimal transformation field by evaluating the similarity metrics at each step. Generally speaking, current popular strategies can be classified into two categories: function value based, e.g., Powell's method, and gradient based, e.g., steepest descent or conjugate gradient. In practice, those strategies are tailored to fit in certain specific circumstances, in order to either speed up convergence, or to avoid local minima.

\subsubsection{Registration as an Ill-Posed Problem}

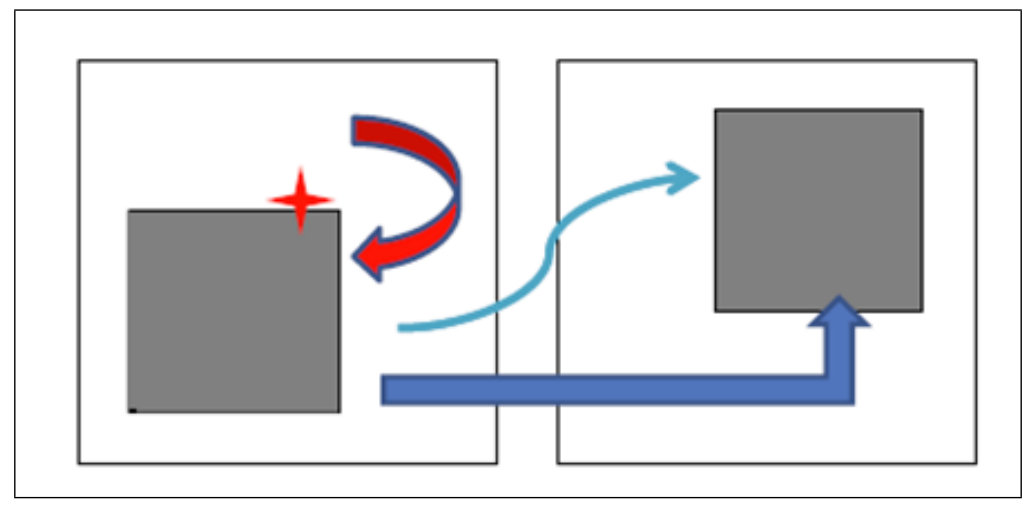

Figure 1.3: Infinite solutions for image registration

When formulated with the similarity metrics alone, image registration is an inherently ill-posed [21] problem with infinitely many solutions for a pair of inputs. Figure 1.3 presents a very simple example, showing a gray square on a white background. The possible transformations between them are limitless: translation firstly along the $X$-axis 
direction, then $Y$-axis direction; rotation about 180 degrees around the center of the image; or even winding path from the left-bottom to right-top corner. This example indicates that a mathematically valid solution to the optimization problem may not be accepted as legitimate under the context of image registration, where unique and meaningful transformations are highly desired in real world applications, especially in the medical field. Consequently, regularization for image registration is necessary in order to produce meaningful matching and integrate users' (e.g., medical practitioner) domain knowledge into the framework. Thus, image registration can be further described as a constrained optimization problem, of which the objective function consists not only of the similarity metrics $D$, but also certain soft constraints (penalty) and/or hard constraints (guarantee).

The implementation of soft constraints is to add a penalty term Reg to the objective function, penalizing unwanted solutions. The overall framework would be:

$$
\text { Minimize: } \quad J(T)=D(R, F(T))+\lambda \operatorname{Reg}(T)
$$

where $\lambda$ is a weighting factor that controls the relative influence of similarity metrics and regularization. Automatically setting up the optimal value of $\lambda$ is a nontrivial issue: too large $\lambda$ will effectively rule out the unsatisfied solutions, however, probably resulting in poor registration effect; too small $\lambda$ will not guarantee the uniqueness of the transformation in spite of good matching.

In contrast to the penalty approach, approaches based on hard constraints can completely rule out the unwanted transformations, thus ensuring the uniqueness of the registration results. The formulation for this approach is usually as below:

Minimize: $J(T)=D(R, F(T))$

Subject to: $C(T)$ 
where the term $C(T)$ is the constraint condition, which could be either an inequality or equality equation that describes a specific regularization enforced for different purposes.

Besides the explicit approaches, a group of solutions rely on implicit model of transformation to achieve regularizations. For example, rigid or affine transformation is internally smooth. Through these regulating constraints, different aspects of regularization can be achieved. A detailed literature review of the related regularization approaches will be given in Chapter 2.

\subsection{Motivation for our Regularity-Guaranteed Image Registration}

Image registration has been successfully employed in various medical applications, greatly advancing the development of diagnostic methodologies. Along with the growing maturity and increasing sophistication of medical imaging techniques, the subjects of study have also become increasingly complex: from simplex tissues (e.g., bone) to organs (e.g., human brain), and from anatomical structures to functional activities.

As the most anagogic organ in the human body, the brain is extremely delicate. It is an essential organ that directly determines the quality of everyone's daily life. Each year, more than 50 million Americans are plagued with neurological illnesses, at costs exceeding $\$ 460$ billion [22]. Neurodegenerative diseases, including Alzheimer's Disease (AD), Parkinson's Disease (PD), etc, are among the most studied neurological abnormalities across the world. Continuous brain tissue deformation is one of the common patterns among these diseases, which gradually leads to various deteriorations in neurological functions. Longitudinal neuroimaging studies conducted on different groups have helped reveal the development paths in $\mathrm{AD}$ and $\mathrm{PD}$, where the correspondence obtained through image registration is an essential component to quantitatively observe the changes over time.

Although the importance of the role image registration plays in longitudinal studies is well recognized, the specific regularization regarding the unique characteristics of 
neurodegenerative diseases has not been extensively researched. When it comes to registering human brain images, we believe two particular requirements should be put as the first and foremost considerations: topology preservation and registration accuracy. The reasons are multifold. For neurodegenerative diseases, especially AD, the shrinkage and expansion in various brain areas are gradual and continuous, where the topological relationship among different cortical and subcortical structures is relatively well maintained, at least in the early stage of the disease. In order to recover meaningful correspondence and deformations over time, topology preserving image registrations that guarantee the regularity of the estimated deformation fields are highly desired.

Accurately capturing disease progression process is another vital component of a reliable longitudinal study. The Jacobian determinants (details will be given in chapter 2) of the estimated deformation fields provide a biologically interpretable measure of local volumetric expansion or compression. After removing the global effects of size, the combined voxelsize Jacobian can be used to reveal certain global variations among different, e.g, age or gender, population groups. This is the foundation of a very popular quantitative image analysis technique called Deformation-Based Morphometry (DBM) [16].

As a summary, designing topology preservation and regularity guaranteed registration frameworks is highly desirable for conducting longitudinal neuroimaging studies, which is the focus of this thesis.

\subsection{Thesis Contribution}

In this thesis, we review the current regularization methods associated with medical image registration, and develop a novel and robust regularization method for easily integrating prior knowledge about neurodegenerative diseases into an efficient constrained optimization framework. The major contributions can be summarized as: 
- An extensive and comprehensive literature review regarding the regularization methods in medical image registration is summarized;

- A novel and robust regularization method is developed and validated;

- In contrast to previous work, we point out and differentiate the Convex/Concave transformation cases;

- An efficient constrained optimization framework based on the Augmented Lagrangian Multiplier method is carried out under two representative transformation models.

\subsection{Thesis Outline}

The rest of the thesis is organized as follows: Chapter 2 will give a comprehensive literature review of the regularization involved in image registration, as well as the specific requirement for neurodegenerative diseases. In Chapter 3, the details of our novel regularity-guaranteed method will be described, and the corresponding registration component of the framework will also be examined, including the detailed numerical solution used in this thesis. In Chapter 4, experimental results using our model are presented, together with the comparisons made with other models. Chapter 6 concludes this thesis and lays out the steps to complete the future work. 


\section{ReLATED WORK}

In this chapter, we will conduct an extensive literature review of regularization methods in medical image registration. The advantages and limitations of each method will be closely examined, followed by the specific requirements of neurodegenerative diseases (subjects of interest in this thesis) for topology regularization.

\subsection{Regularization in Image Registration}

It has been realized for a long time that image registration is an inherently ill-posed problem [21]. Regularization constraints are needed to limit the number of possible solutions. The existing regularization methods can be roughly classified into two classes: regularity constraints on transformation smoothness and on transformation topology.

Those two types of regularizations have been extensively researched. To smoothe the transformation, either basis function based, or physical model based methods can be utilized; to preserve the topology, the majority of current work mainly relies on limiting the Jacobian, which will be illustrated in section 2.1.2.

\subsubsection{Regularization on Smoothness}

The transformation field sought for during the image registration is firstly required to be smooth enough, when the deformation type for objects of interest in images has been analyzed from a global scale to a local one. Strictly speaking, the mathematical definition of a "smooth" transformation field means it is differentiable, while in practice, this condition may be compromised due to either a soft constraint, or computational expense. Broadly, the smoothness can be imposed into transformation field by two ways, basis function expansion based, and physical model based. 


\subsubsection{Basis Function Expansion based}

As explained in section 1.1, the transformation field $T(X)$ should be applied to each pixel of the images, while basis function expansion based transformation models use a set of basis functions, the coefficients of which are adjusted so that the combinations of them can fit the transformation field. The majority of these models are stemming from the theory of function interpolation [32] and approximation [38, 42]. As a result, the approximation function is usually much smoother than the originally dense transformation field. Several well-used basis function expansion based models are introduced as following:

\section{Global transformation [48]}

Rigid: a transformation model is called rigid, only when the relative distances among points within the images are preserved. This means there are just translations and rotations during the transformation, which is described as:

$$
\left\{\begin{array}{c}
u(\mathrm{x}, \mathrm{y})=\left(\cos (\phi) x-\sin (\phi) y+d_{x}\right)-x \\
v(x, y)=\left(\sin (\phi) x+\cos (\phi) y+d_{y}\right)-y
\end{array}\right.
$$

where $u(x, y)$ and $v(x, y)$ denote the displacement at point $(x, y)$ along the $X$-axis and $Y$-axis direction. $\phi$ stands for the rotation angel, and $\left(d_{x}, d_{y}\right)$ is the translation vector.

Affine: an affine transformation model can preserve the parallelism of two lines, which can be expressed as:

$$
\left\{\begin{array}{c}
u(\mathrm{x}, \mathrm{y})=\left(\mathrm{a}_{11} x+a_{12} y+d_{x}\right)-x \\
v(x, y)=\left(a_{21} x+a_{22} y+d_{y}\right)-y
\end{array}\right.
$$

where

$$
\left(\begin{array}{ll}
a_{11} & a_{12} \\
a_{21} & a_{22}
\end{array}\right)
$$


is called the affine matrix, specifies the scaling and shearing changes of the transformation.

Those global transformation models are always used in the first step before more refined non-rigid models during registration, so that the local minimum can be avoided, and the optimization can be sped up.

\section{Radial basis function [48]}

A radial basis function is an univariate continuous function of the distance $\left\|x-x_{i}\right\|$ between the interpolation point $x$ and the basis function centers (or control points, land positions) $x_{i}$. A standard radial basis function is defined as:

$$
T(x)=\sum_{i}^{n} \omega_{i} \phi\left(\left\|x-x_{i}\right\|\right)
$$

where $i$ indexes the control points, $n$ is the total number used, and $\omega$ are weights determined by solving a set of linear equations. $\phi$ is standing for different forms of radial basis functions, such as multiquadrics, weighed mean, thin-plate splines (TPS), etc.

Multiquadrics: in multiquadric form, the $\phi\left(\left\|x-x_{i}\right\|\right)$ is defined as follows [10]:

$$
\phi\left(\left\|x-x_{i}\right\|=\sqrt{r_{i}^{2}+d^{2}}\right.
$$

where $r_{i}$ is the Euclidean distance. The parameter $d$ controls the degree of smoothing, with larger $d$ leading to stronger smoothing.

Weighed mean: in weighted mean, the $\phi\left(\left\|x-x_{i}\right\|\right)$ is defined as:

$$
\phi\left(\left\|x-x_{i}\right\|=\frac{G_{i}\left(\|\left|x-x_{i}\right|\right)}{\sum_{i}^{n} G_{i}\left(\left\|x-x_{i}\right\|\right)}\right.
$$


where $G_{i}$ stands for a monotonically decreasing radial basis function, such as Gaussian or Cubic $G_{i}$.

TPS: TPS was originally used to design structures such as aircraft wings, and was then applied to functions and interpolations $[6,25]$. The $\phi\left(\left\|x-x_{i}\right\|\right)$ here is defined as:

$$
\phi\left(\left\|x-x_{i}\right\|=\sum_{i=1}^{n} F_{i} r_{i}^{2} \ln r_{i}^{2}\right.
$$

\section{B-splines}

Originally proposed by Schoenberg [41], B-splines were often used to interpolate and approximate scattered data. Recently, several papers, [30, 40] argued that B-splines are optimal as approximating functions that can be used to model deformations in registration. The most common form of $\mathrm{B}$-splines used in registration is the cubic B-spline, which is $C^{2}$ continuous. For a control point $P_{i, j, k}(3 \mathrm{D})$ in a grid $P$ $(i=-1,0, \ldots, l+1, j=-1,0, \ldots, m+1$, and $k=-1,0, \ldots, n+1)$, the transformation field (3D) is calculated as:

$$
T(x, y, z)=\sum_{l=0}^{3} \sum_{m=0}^{3} \sum_{n=0}^{3} B_{l}(u) B_{m}(v) B_{n}(w) \phi_{i+l} \phi_{j+m} \phi_{k+n}
$$

where $B$ are the coefficient functions:

$$
\begin{aligned}
& B_{0}(u)=\frac{\left(1-u^{3}\right)}{6} \\
& B_{1}(u)=\frac{\left(3 u^{3}-6 u^{2}+4\right)}{6} \\
& B_{2}(u)=\frac{\left(-3 u^{3}+3 u^{2}+3 u+1\right)}{6} \\
& B_{3}(u)=\frac{u^{3}}{6}
\end{aligned}
$$


The B-splines basis functions can be extended to multivariate ones using tensor products, of which FFD-based B-spline is a very common example [12].

\section{DCT/DST}

Mathematically, a discrete cosine/sine transform (DCT/DST) expresses a sequence of finitely many data points in terms of a sum of cosine/sine functions oscillating at different frequencies [3]. A set of low frequency 2D DCT basis functions are given in Figure 2.1. The choice between DCT and DST usually relies on the restriction of the image boundaries: if points at the image boundaries are required to be stable during the registration, DST are selected; if no constraints are imposed on the boundaries, DCT is preferable. Here, we would just introduce DCT.

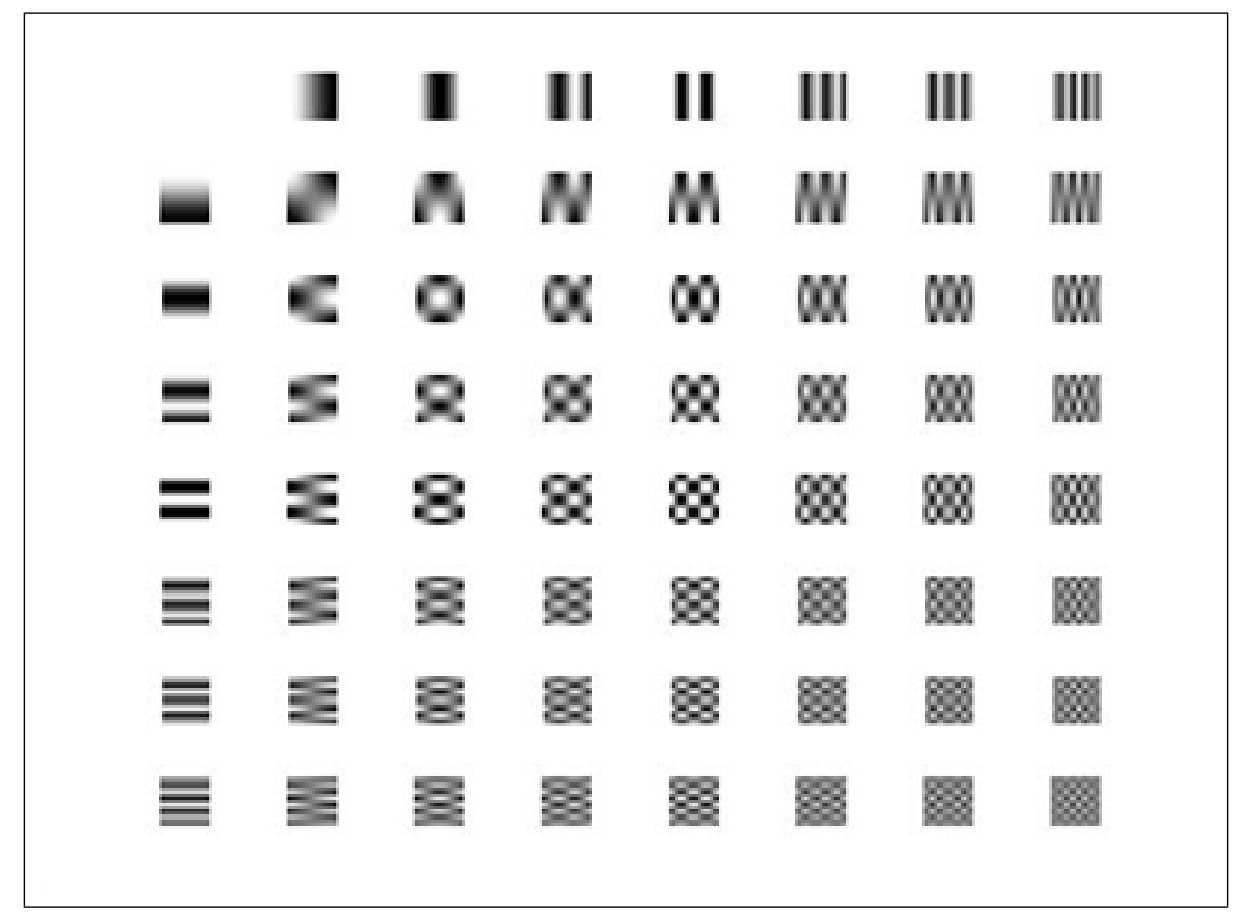

Figure 2.1: The lowest frequency basis functions of 2D DCT 
In one dimension, the DCT of a function is generated by pre-multiplication with the matrix $D^{T}$, where the elements of the $M$ by $N$ matrix $D$ are defined by:

$$
\begin{aligned}
& d_{m, 1}=\frac{1}{\sqrt{M}} \quad m=1 \ldots M \\
& d_{m, n}=\sqrt{\frac{2}{M}} \cos \left(\frac{\pi(2 m-1)(n-1)}{2 M}\right) \quad m=1 \ldots M, n=1 \ldots N .
\end{aligned}
$$

In two dimensions, the DCT of a $I$ by $J$ matrix $A$ is generated by the production:

$$
A=D_{1} * Q * D_{2}^{T}
$$

where $D_{1}$ is a $I$ by $M$ matrix, which can be obtained using the equation 2.9 ; and $D_{2}$ is a $J$ by $N$ matrix, which can also be obtained in the same way. And, $Q$ is a $M$ by $N$ coefficient matrix. Consequently, if $T(x)$ is considered as two $I$ by $J$ matrices $T_{x}(x)$ and $T_{y}(x)$ respectively for $X$-axis and $Y$-axis direction, the transformation field would be constructed by $D_{1} * Q_{1} * D_{2}^{T}$ and $D_{1} * Q_{2} * D_{2}^{T}$. Therefore, the optimization process would seek the appropriate coefficient matrices $Q_{1}$ and $Q_{2}$, which, in the end, $T(x)$ can be easily calculated.

\subsubsection{Physical Model based}

Another group of regularization models are formulated under certain physical laws. With various materials as the assumed underlying medium, different levels of regularization smoothness can be achieved.

\section{Linear elastic model}

Linear elastic model is derived from the theory of linear elasticity based on notions of stress and strain [44]. Generally speaking, when a body is subject to an external force, this would lead to internal forces within it, causing deformation. The internal forces are classified into body and surface forces. When they are balanced with each 
other, a linear elastic material is in equilibrium, which could be described by the Navier-Cauchy linear elastic equation [44]:

$$
\mu \nabla^{2} \vec{u}+(\mu+\lambda) \nabla(\nabla \cdot \vec{u})+\vec{f}=0
$$

where $\mu$ and $\lambda$ are Lame parameters [20], $u$ is the displacement field, the change of transformation at each step $(\Delta T)$. And $f$ denotes the body force, here, standing for the driving force for registration (numerically, the first order derivative of the cost function). To implement this model, one has to solve the elastic linear equation 2.11 to obtain the update of transformation field $u$ at each step, until the similarity metrics are minimized.

This linear elastic model based on the Navier-Cauchy equation is essentially an optimization process involving the balance of the external force and the internal stress, thus imposing smoothness on the update of transformation. This model can be integrated with different methods, such as variational frame [33], finite difference [4, 8], FEM [24], and Fourier transform method [32]. Due to the restrictive linear elasticity, this model may not be plausible for many biological materials that have a non-linear stress-strain relationship. Meanwhile, it is only accurate for small deformations.

\section{Viscous fluid flow model}

Viscous fluid flow model was proposed to recover large deformations, which cannot be done by linear elastic model that relies on the assumption of an infinitesimal small deformation [15]. The underlying foundation for the viscous fluid model is continuum mechanics, which brings up physical laws conforming to Newtonian mechanics, such as the conservations of mass, energy, and linear and angular momentum. To put it simply, in contrast to the linear elastic model that is characterized by a spatial smoothing of the transformation change $u$, the viscous fluid 
model is characterized by a spatial smoothing of a special fluid term - fluid velocity $v$, which can be obtained through:

$$
v(x, t)=\frac{\partial u}{\partial t}+v \nabla u
$$

Similarly like the Navier-Cauchy equation, the Navier-Stokes equation describes the equilibrium state of a given region of the fluid, where the changes in momentum balance with the changes in pressure and dissipative viscous forces, as expressed:

$$
\mu \nabla^{2} v+(\mu+\lambda) \nabla(\nabla \cdot v)+f=0
$$

in which, $\lambda$ and $\mu$ are viscosity constants. At each time step, the velocity field is calculated in viscous fluid registration, rather than the transformation field in elastic registration. This model was firstly proposed by Christesen et al. [13-15] to deal with the large deformation in biomedical materials. Although it allows large local deformation, solving the Navier-Stokes equation is computationally intensive, which is a major problem.

\section{Optical flow model}

Optical flow is the pattern of apparent motion of objects, surfaces, and edges in a visual scene caused by the relative motion between an observer and the scene $[11,47]$. The basis hypothesis of the optical flow model is based on the intensity conservation between image frames, which can be described as:

$$
\vec{u} \cdot \vec{\nabla} s=m-s
$$

where $m$ and $s$ are intensity for two image frames, which, in registration, could be regarded as floating and reference images separately; $u$ is still the displacement field. 
Thirion [45] was inspired by this, and considered the non-rigid registration as a diffusion process, bringing up the well known demons algorithm. He introduced demons that push according to local property of images, in a similar way as Maxwell did for solving Gibbs paradox. The displacement is calculated through a numerical stable expression of the equation 2.14 , as follows:

$$
u=\frac{(m-s) \nabla s}{\|\nabla s\|^{2}+(m-s)^{2}} .
$$

Meanwhile, to reduce the effects of noise, the displacement field $u$ is smoothed by Gaussian convolution. During the whole algorithm, the displacement at each iteration is calculated by the equation 2.15 , and the reference image is refreshed for the next iteration.

\subsubsection{Regularization on Topology}

Comparing with regularization on smoothness, topology preservation is a hard and more localized constraint, which ensures that connected structures remain connected, and the relative positions among different structures are well maintained over the deformation process. In longitudinal studies, topology stability among different brain structures/tissues is expected for individual subjects. Correspondingly, topology preservation makes a meaningful and justified requirement, and any reasonable deformation estimation algorithm should conform to it.

The property of topology preservation is highly related to the continuity and inversibility of the transformation field. Mathematically, to be a topology-preserving transformation, $T(x)$ must be: (a) continuous, (b) bijective (one on one) mapping [34]. The continuity of the transformation is to ensure the local neighborhood relationship, while the bijectivity maintains that each point in the floating image has one and only one corresponding point in the reference image and conversely. 
To meet the greater challenges of observing and tracking biological deformation, a lot of work has been done to better preserve topology of transformation in image registration $[15,26,27,34,35,39,43]$. Currently, almost all of the topology-preserving approaches are based on the Jacobian matrix and its determinants.

The Jacobian matrix is consisting of all first-order partial derivatives of a vectoror scalar-valued function with respect to another vector. In the application of image registration, it is applied to the transformation field in each direction, which is described as followed:

$$
\mathbf{J}=\left(\begin{array}{cc}
\frac{\partial T_{x}(x)}{\partial x} & \frac{\partial T_{x}(x)}{\partial y} \\
\frac{\partial T_{y}(x)}{\partial x} & \frac{\partial T_{y}(x)}{\partial y}
\end{array}\right)
$$

where $T_{x}(x)$ and $T_{y}(x)$ are the Cartesian components of the transformation field $T(x)$ over $X$-axis and $Y$-axis direction. And, its determinant $J_{T}$ is also simply called the Jacobian.

The reason for using the Jacobian is its good preservation of local bijectivity, which is obtained by enforcing $J_{T}$ to be positive all over the transformation domain. Furthermore, the transformation is incompressible if $J_{T}$ is equal to one, locally compressible if it is less than one, and expansible if greater than one.

Christensen et al. [15] firstly embedded the topology-preserving regularity into a viscous fluid material deformation model, under an Eulerian framework. The NavierStokes partial derivative equations were used to deal with large deformation. The global positivity of the Jacobian were ensured in this way: when the magnitude of the Jacobian drops below a certain value, specifically 0.5 , the current computation is stopped and a new propagated template image is generated equal to the deformed template at the previous instant. Generally speaking, this could be the first time that topology-preservation is taken into account of during the non-rigid image registration. However, it was not fully illustrated, which, instead, was regarded as the part of smoothing regularity. Meanwhile, 
both the computational cost and the necessity to tract the discrete Jacobian in order to avoid numerical divergence are serious problems that we can't afford to ignore.

In [34] and [35], Armspach et al. proposed a hierarchical model based on B-spline basis functions, which ensures a topology-preserving deformable mapping in both $2 \mathrm{D}$ and 3D space. The topology is preserved by limiting the Jacobian in a positive interval through a penalty approach. And also, different intervals are analyzed to achieve the best regularity effect. They clearly elaborated the definition and significance of topology-preservation in medical image analysis. Nevertheless, their characterization of the Jacobian is valid only with using the linear interpolating spline.

Especially inspired by the case of pre- and post-contrast MR breast images, Rohlfing et al. [39] made the assumption that soft tissue is incompressible for small deformations and short time periods. With a mutual information objective cost function, they penalized it by adding the absolute value of the log of the Jacobian. Correspondingly, a soft constraint that regularizes the local volume-preservation is implemented. Blended together with a smoothness constraint within the overall objective function, the impressibility property is favored, but not guaranteed.

Karacali and Davatzikos [27] also impose enforcement of the constraint based on the Jacobian, which is through a general formalism derived with regard to the discrete approximations to the Jacobian matrix. Although it looses the regularity control, allowing volumetric change within a prescribed interval, hyper regularity is still existing due to preventing the concave deformation.

In [26], Haber et al. set upper and lower bounds for the Jacobian during the registration procedure. A log-barrier function scheme is employed to convert the constraints to an unconstrained optimization problem, where Gauss-Newton approximation is applied to solve the system. Along with a new type of discretization, this method successfully prevents large scale changes of the volume. On the other hand, they have to pay extra 
attention to the discretization of the constraint built on a dense transformation, which is highly computationally unattractive.

Sdika [43] used B-spline as the global smoothness constraint. The topology regularity is imposed through the positivity of the Jacobian, as well as bounded Jacobian derivatives. Meanwhile, instead of the soft constraint used in previous research, he implemented the augmented Lagrangian Multiplier method to solve the constrained optimization problem.

Generally speaking, these works and their variants have one thing in common: the enforced regularity cases specified by the Jacobian constraints are all convex, where the concave cases, allowed in many applications, have been ruled out. Detailed explanation of the convex/concave regularity cases will be given in Chapter 3 .

\subsection{Specific Requirements for Neurodegenerative Diseases}

The human brain, though just a spongy, three-pound mass of fatty tissue, is much more complicated and delicate than what we can imagine. It controls a person's body activities, ranging from heart beat and sexual function to emotion, learning and memory. It is the single most important organ that shapes our thoughts, hopes and characters, which ultimately defines everyone's personality.

However, neurodegenerative diseases, resulting in the progressive loss of structure

or function of neurons in the human brain, devastate its normality badly. Many neurodegenerative diseases including Parkinson's, Alzheimer's, and Huntington's largely affect the population, e.g., more than 50 million Americans annually, at costs exceeding $\$ 460$ billion [22]. Details are given in Table 2.1.

In spite of great efforts poured into this field, robust, safe and effective therapy is still unavailable at present, while great progress has been made in diagnosis through genetics, biochemistry, cell biology, experimental treatments, and brain imaging studies. At present, the final causes and mechanisms are not yet fully understood, but the opinion 
Table 2.1: The Toll of Selected Brain and Nervous System Disorder on Americans

\begin{tabular}{ccc}
\hline \hline Condition & Total Cases & Cost per Year (U.S. dollars) \\
\hline Sleep Disorders & 70 million & 100 billion \\
Alzheimer's Disease & 5 million & 148 billion \\
Parkinson's Disease & 1 million & 5.6 billion \\
Stroke & 5.2 million & 51 billion \\
$\ldots$ & $\ldots$ & $\ldots$ \\
\hline
\end{tabular}

that those diseases are mainly associated with the accumulation of protein degradation has been confirmed. Different diseases may enter different protein degradation pathways at different times, but the commonality among them is the interdependent cell deaths triggered [7]. Reflected into a large-scale and longitudinal analysis, different parts of the human brains are undergoing a dramatic morphologic deformation. Therefore, quantifying the deformation through the medical image analysis would be of great significance in hunting important bio-markers, e.g., deformation starting position, volume change, and change rate. Moreover, the deformation is non-homogenous (the deformation cannot be easily separated into expansion or compression), as shown in Figure 2.2. Obviously, the tissue in the areas of cortex and hippocampus are extremely shrunk, while a large expansion happens in the part of ventricles. Thus, the homogenous regularity with respect to volume change is not suitable.

As stated before, the current topology-preserving works are mainly limiting the Jacobian to certain values or between certain intervals, which is homogenous with respect to the volume change. After fully considering the special case in neurodegenerative diseases, we claim that it is more essential to keep the relative neighborhood relationship between the tissue cells in the topology preservation of the human brain, rather than to 


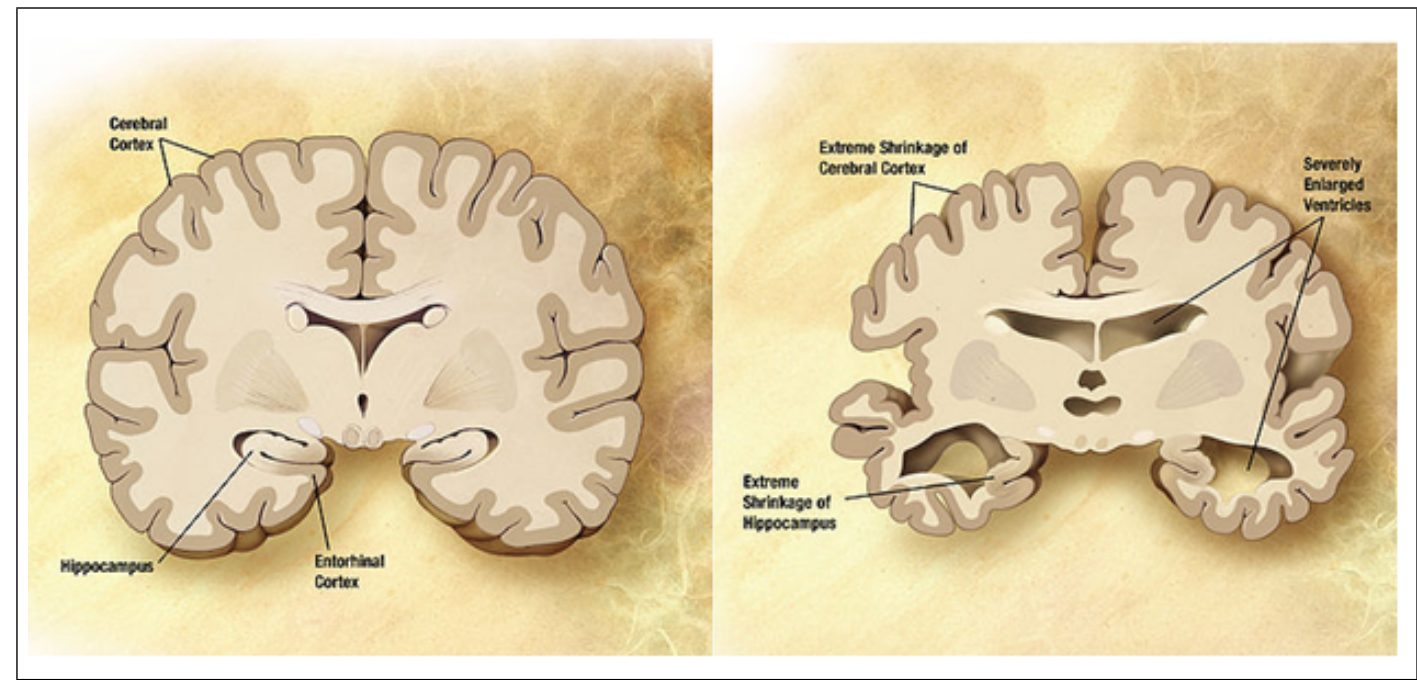

Figure 2.2: Comparison of a normal aged brain (left) and an Alzheimer's patient's brain (right) (from the webpage of "Alzheimer's Disease Education and Referral Center, a service of the National Institute on Aging.").

constrain the whole volume. Inspired by this, we propose a new Non-Twist regularization based on point-line/surface orientation theory, which guarantees the relative position of each pixel or voxel in registration, thus preserving the topology. 


\section{Non-Twist Regularization Framework}

In this chapter, we introduce our Non-Twist guaranteed image registration in detail. Our Non-Twist regularization is fully illustrated from the original derivation of point-line

orientation theory. Besides, the four crucial components of image registration framework used in this thesis are also explained.

\subsection{Non-Twist Regularization}

Topology regularity, more specifically the preserved neighborhood relationship, will be violated if transformation grid corners flip their relative positions during the registration procedure. Shown in Figure 3.1 are a 2D grid and the associated transformation scenarios. Let $A$ be the point of interest, and $A B C D$ be the grid to study. After each spatial update, A might end up in one of the three destination areas in Figure 3.1(a), marked with gray, orange, and blue colors, respectively. The corresponding resultant grids are shown in Figure 3.1 (b), (c) and (d). Because it satisfies the positivity of the Jacobian criterion, scenario (b) is usually taken as a topology preservation case, and we call it the convex case, as it maintains the convexity of the starting grid $A B C D$. Scenario (c), which we call the concave cases, are regarded as illegal and ruled out by most aforementioned works, even though they are acceptable in reality as no twisting has happened. Cases in (d) are indeed twisting the grids and they should be prevented from happening.

Identifying the three cases (convex and concave, and twisting) can be easily performed through the point-line/surface test [19] commonly used in the computational geometry community, which is illustrated in Figure 3.2. 


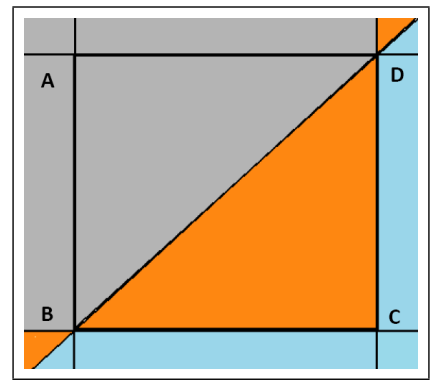

(a)

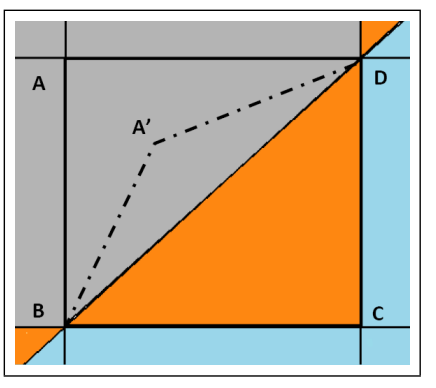

(b)

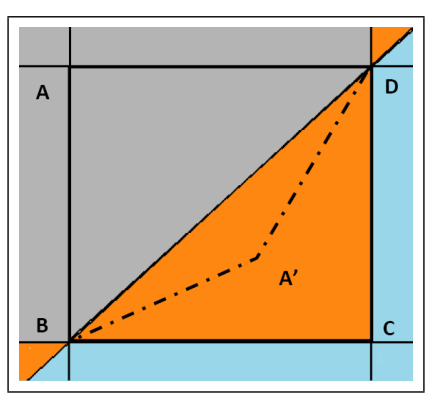

(c)

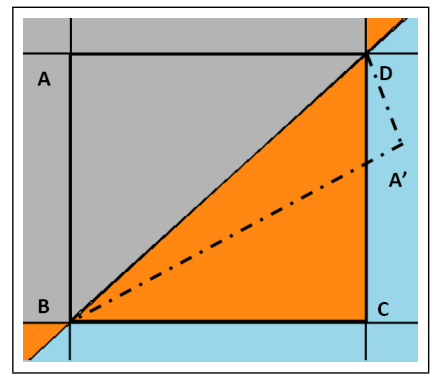

(d)

Figure 3.1: Illustration of possible grid update scenarios. (a) starting grid; (b) convex case; (c) concave case; (d) twisting case, which should be avoided.

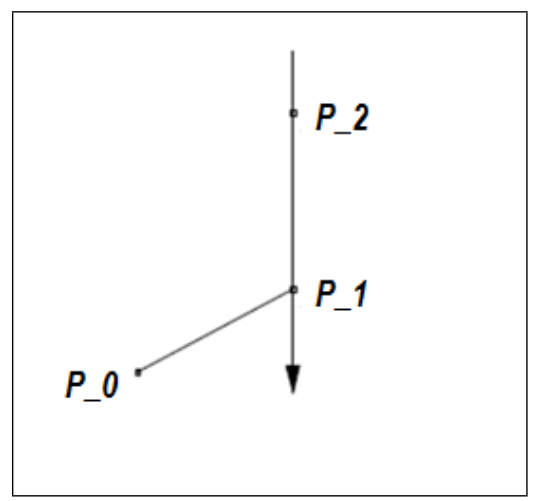

(a)

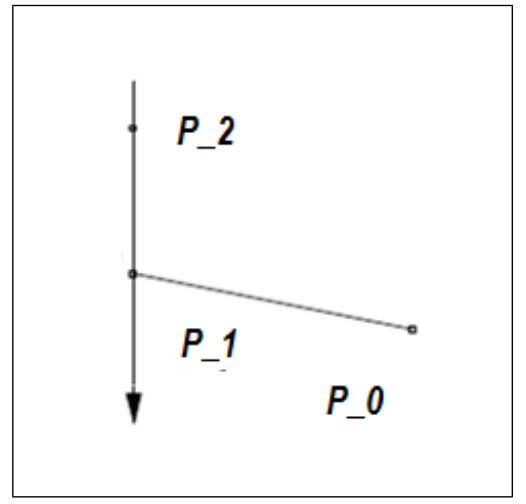

(b)

Figure 3.2: Point-line orientation. (a) Positive orientation: $P 0$ is on the right side of the line $P 2 P 1$; (b) Negative orientation: $P 0$ is on the left side of the line $P 2 P 1$. 
In Figure 3.2, two point-line orientations are given. To decide the orientation numerically, we use the matrix:

$$
\boldsymbol{\Lambda}=\left(\begin{array}{lll}
X_{0} & Y_{0} & 1 \\
X_{1} & Y_{1} & 1 \\
X_{2} & Y_{2} & 1
\end{array}\right)
$$

$X$ and $Y$ are coordinates for each point, and the order of the points should be paid attention to. The orientation of $P_{0}$ to $\overrightarrow{P_{2} P_{1}}$ is positive if and only if sign $(\operatorname{det} \Lambda)=+1$, and it is negative if and only if $\operatorname{sign}(\operatorname{det} \Lambda)=-1$.

Based on this, the convex case requires a combination of three negative orientations: $A$ is on the left side of $\overrightarrow{B D}$, left side of $\overrightarrow{B C}$ and left side of $\overrightarrow{C D}$. Converting to matrix format, the following three constraints have to be held to ensure the convex case.

$$
\left|\begin{array}{lll}
x_{a} & y_{a} & 1 \\
x_{d} & y_{d} & 1 \\
x_{b} & y_{b} & 1
\end{array}\right|<0, \quad\left|\begin{array}{lll}
x_{a} & y_{a} & 1 \\
x_{c} & y_{c} & 1 \\
x_{b} & y_{b} & 1
\end{array}\right|<0, \quad\left|\begin{array}{lll}
x_{a} & y_{a} & 1 \\
x_{d} & y_{d} & 1 \\
x_{c} & y_{c} & 1
\end{array}\right|<0 .
$$

Combing the convex and concave cases together (the gray and orange areas in Figure 3.1), the overall accepted update scenarios can be translated to a Boolean predicate: ( $A$ is on the left side of $\overrightarrow{B D}$ ) $\mathbf{O R}$ ( $(A$ is on the left side of $\overrightarrow{B C}$ ) AND ( $A$ is on the left side of $C D)$ ).

Furthermore, if this regularity is applied to each corner of each transformation grid during the whole iteration process, no twist would happen in each step, thus maintaining the relative neighborhood relationship.

\subsection{Integration with Registration Framework}

Our Non-Twist regularization can be easily integrated into various registration algorithms. In this thesis, we choose the Demon's algorithm and the DCT-based algorithm (used in SPM [23]) as the testbed to show the effectiveness of our constraint. Justification of 
using these two algorithms lies in the fact that they belong to different algorithm categories (physical model based and radial basis function based), and both have been widely used across the neuroimage community. Like many other non-rigid registration algorithms, the Demon's and the DCT are also formulated as the optimization of an objective function to determine transformation field $T$ that minimizes the difference between the reference image $R$ and floating image $F$. the Demon's uses Sum of Squared Differences (SSD) as the similarity metric, and the squared gradient of the transformation field as smoothness regularization. Given the transformation field $T$, it computes a correspondence update displacement field $u$ by minimizing:

$$
E(u)=|R-F \cdot(T+u)|^{2}+\sigma^{2}|u|^{2}
$$

where $\sigma$ is a constant for intensity and transformation uncertainty.

The DCT-algorithm also uses SSD as the similarity metric. Unlike in the Demon's, the smoothness property in the DCT, however, is fulfilled through a linear combination of lower-frequency components of the DCT. The deformation field can be obtained by solving a small set of coefficients controlling the basis functions. For more details with respect to these two methods, we refer readers to Chapter 2.

Integrating our Non-Twist constraint with the Demon's and the DCT is straightforward. Taking the convex case as an example, the new optimization objective is simply:

$$
\begin{array}{ll}
\text { Minimize: } & E(u) \\
\text { Subject to: } & (A \Uparrow B D) \text { AND }(A \Uparrow B C) \text { AND }(A \Uparrow C D)
\end{array}
$$

where $A \Uparrow B D$ denotes the requirement that $A$ lies on the left side of $\overrightarrow{B D}$. 


\subsection{Numerical Solutions for Constrained Optimization Problem}

As illustrated in a previous section, our proposed Non-Twist constrained image registration has been reduced to a typical constrained optimization problem, whose standard form is as follows:

$$
\begin{array}{ll}
\text { Minimize: } & f(x) \\
\text { subject to: } & \begin{cases}g_{i}(x) \leq 0, & i=1, \cdots, m \\
h_{j}(x)=0, & j=1, \cdots, l\end{cases}
\end{array}
$$

where $f(x)$ is the objective function to be minimized, $g(x)$ are a set of inequality constraints, and $h(x)$ are a set of equality constraints. Previous approaches based on the Jacobian usually use inequality constraints to limit the value of the Jacobian in certain intervals, while our approach takes use of equality constraints to restrain the sign of the Boolean predicates 3.4 to be either 1 or -1 .

\subsubsection{Constrained Optimization}

In Chapter 1, we have mentioned that optimization constraints can also be divided into soft or hard types from the algorithmic point of view. Hard constraints are the ones that must be satisfied at all optimization iterations, and must be feasible at all iterations; soft constraints are not so strict: they require that the final results are indeed the minimizer, but during the process, the constraints may be sacrificed to find the shortcut. Consequently, feasible algorithms using hard constraints are usually slower and more computationally expensive, and cannot follow shortcuts to the solution that crosses the infeasible territory [36].

To solve the constrained nonlinear optimization, the Karush-Kuhn-Tucker (also known as the Kuhn-Tucker or KKT conditions) are necessary for a solution to be optimal, given that some regularity conditions (KKT conditions) are satisfied [28, 29]. Recall 
the optimization problem in 3.5 , where the objective function $f(x)$ is: $R^{n} \rightarrow R$ and the constraints are $g_{i}: R^{n} \rightarrow R$ and $h_{i}: R^{n} \rightarrow R$. Meanwhile, they have to be continuously differentiable at a point $x^{*}$. The KKT conditions claim that if $x^{*}$ is a local minimum that satisfies some regularity conditions, then there exist constants $\mu_{i}(i=1, \ldots, m)$ and $\lambda_{j}(j=1, \ldots, l)$ ), called KKT multipliers (or Lagrange multipliers), such that the following properties will be valid [2]:

Stationarity:

$$
\nabla f\left(x^{*}\right)+\sum_{i=1}^{m} \mu_{i} \nabla g_{i}\left(x^{*}\right)+\sum_{j=1}^{l} \lambda_{j} \nabla h_{j}\left(x^{*}\right)=0
$$

Primal feasibility:

$$
\begin{aligned}
& g_{i}(x *) \leq 0, \quad \text { for all } i=1, \ldots, m \\
& h_{j}(x *)=0, \quad \text { for all } j=1, \ldots, l
\end{aligned}
$$

Dual feasibility:

$$
\mu_{i} \geq 0, \quad \text { for all } i=1, \ldots, m
$$

Complementary slackness:

$$
\mu_{i} g_{i}(x *)=0, \quad \text { for all } i=1, \ldots, m
$$

Although KKT conditions may not lead directly to a very efficient and numerical algorithm for Nonlinear Program Problems (NLPs), they do contribute in the following aspects: 
- They give insight into what optimal solutions to NLPs look like;

- They provide a way to set up and solve small problems;

- They provide a method to check solutions to large problems;

- The Lagrange multipliers can be seen as shadow prices of the constraints.

\subsubsection{Practical Numerical Solutions}

Practically, to solve constrained optimization problems, numerous solutions have been proposed, which include but are not limited to: penalty function method, Lagrange Multiplier method, augmented Lagrange Multiplier method, Quadratic programming, etc. In this thesis, to implement this optimization with our proposed constraint and validate its expected effect, in the early stage, a large-scale nonlinear algorithm using interiorpoint based sequential quadratic programming (SQP) is adopted and adjusted specifically. Because of its use of a trust region framework that allows for the direct use of second derivatives and the inaccurate solution of sub-problems [12], this optimization algorithm is comparably efficient in dealing with the dense transformation field. Since the whole problem is large and indefinite, previously, we took use of the currently available function

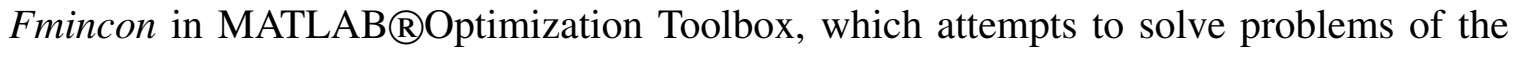
form:

$$
\begin{array}{ll}
\text { Minimize: } & F(X) \\
\text { subject to: } & \left\{\begin{array}{l}
A * X<=B, \text { Aeq } * X=B e q \text { (linear constraints) } \\
C(X)<=0, C e q(X)=0 \text { (nonlinear constraints) } \\
L B<=X<=U B \text { (bounds) }
\end{array}\right.
\end{array}
$$

where $F(x)$ is the objective function, and $X$ is the variable of solution. The constraints include linear, nonlinear, and boundary. To fit our proposed method, we only need to set 
up the nonlinear equality constraint, and satisfy the other constraints by using identical equations or inequations.

The numerical solution of a registration approach largely determines its practical use. Although using the embedded function Fmincon is very convenient to validate our proposed regularization in the early stage, its potential and performance to be applied in $3 \mathrm{D}$ is in doubt. Therefore, implementing the numerical solutions by our own is necessary for solving the constrained optimization problem in $3 \mathrm{D}$.

An important and widely used class of methods for solving constrained optimization problems is to convert the original constrained problem to a sequence of unconstrained subproblems, which includes Quadratic penalty method, Logarithmic barrier method, and augmented Lagrangian method [36]. The fundamental approach of this class is to replace the original problem by a new objective function that consists of [36]

- the original objective of the constrained optimization problem, plus

- additional terms for each constraint, which is positive when the current point $x$ violates that constraint and zero otherwise.

\section{Quadratic penalty method}

The simplest penalty function is the quadratic penalty [36], in which the penalty terms are the squares of the constraint conditions. Here, we only discuss the equalityconstrained optimization. Based on the problem described in the equation 3.5, the new unconstrained quadratic penalty objective function is

$$
Q(x ; \mu)=f(x)+\frac{1}{2 \mu} \sum_{i} h_{i}^{2}(x),
$$

where $\mu>0$ is the penalty parameter. The increasing severity of penalizing the constraint violations is relying on driving $\mu$ to zero. The practical algorithmic framework is specified in Figure 3.3. 


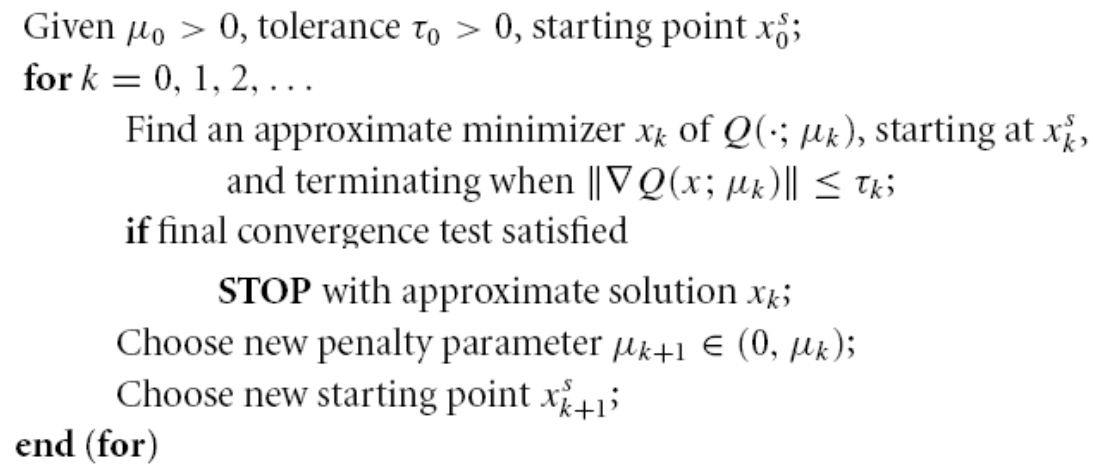

Figure 3.3: Algorithm of the quadratic penalty method

\section{Logarithmic barrier method}

A different approach is the logarithmic barrier method, in which logarithmic terms are used to prevent the optimization path from moving too close to the boundary of the feasible region [36]. The new unconstrained logarithmic objective function is

$$
P(x ; \mu)=f(x)-\mu \sum_{i} \log h_{i}(x),
$$

where log denotes the natural logarithm. The practical algorithmic framework is specified in Figure 3.4.

\section{Augmented Lagrangian method}

Both the quadratic penalty method and logarithmic barrier method are ill conditioning [36], and they are not strictly feasible constrained optimization methods using hard constraints. The augmented Lagrangian method is related to the quadratic penalty method, but it avoids the ill conditioning inherent in this method by introducing explicit Lagrange multiplier estimates at each iteration into the objective function [36]. Also, it tends to yield less ill conditioned subproblems than does the logarith- 


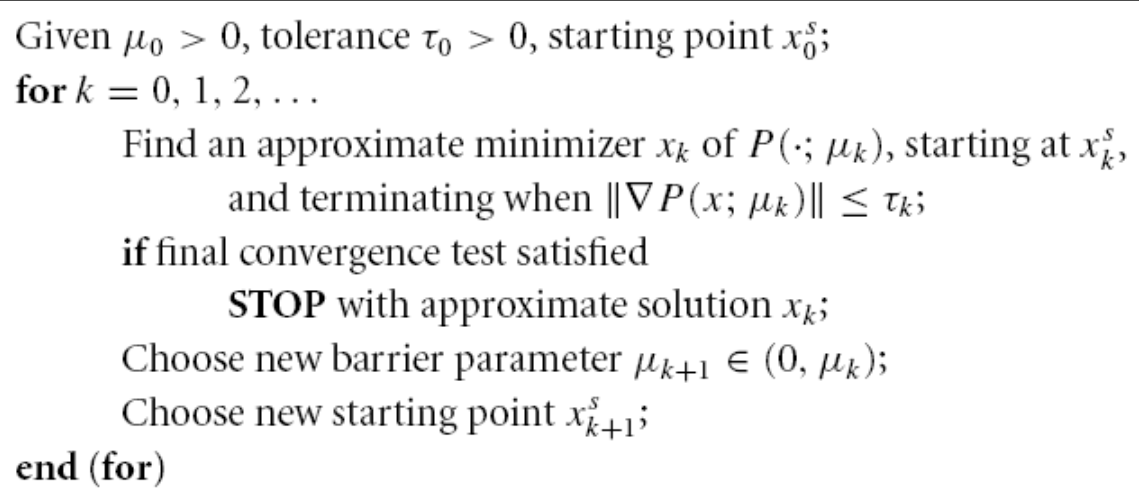

Find an approximate minimizer $x_{k}$ of $P\left(\cdot ; \mu_{k}\right)$, starting at $x_{k}^{s}$, and terminating when $\left\|\nabla P\left(x ; \mu_{k}\right)\right\| \leq \tau_{k} ;$

if final convergence test satisfied

STOP with approximate solution $x_{k}$;

Choose new barrier parameter $\mu_{k+1} \in\left(0, \mu_{k}\right)$; end (for)

Choose new starting point $x_{k+1}^{s}$;

Figure 3.4: Algorithm of the logrithmic barrier method

mic barrier method. Thus, it is a strictly feasible method to maintain hard constraints. The augmented Lagrangian function is

$$
\mathcal{L}(x ; \lambda ; \mu)=f(x)-\sum_{i} \lambda_{i} h_{i}(x)+\frac{1}{2 \mu} \sum_{i} h_{i}^{2}(x),
$$

where $\lambda$ is the Lagrange multipliers based on KKT theory. The practical algorithmic framework is specified in Figure 3.5.

Given $\mu_{0}>0$, tolerance $\tau_{0}>0$, starting points $x_{0}^{s}$ and $\lambda^{0}$;

for $k=0,1,2, \ldots$

Find an approximate minimizer $x_{k}$ of $\mathcal{L}_{A}\left(\cdot, \lambda^{k} ; \mu_{k}\right)$, starting at $x_{k}^{s}$, and terminating when $\left\|\nabla_{x} \mathcal{L}_{A}\left(x, \lambda^{k} ; \mu_{k}\right)\right\| \leq \tau_{k}$;

if final convergence test satisfied

STOP with approximate solution $x_{k}$;

Update Lagrange multipliers using $\lambda_{i}^{k+1}=\lambda_{i}^{k}-c_{i}\left(x_{k}\right) / \mu_{k}$ to obtain $\lambda^{k+1}$; Choose new penalty parameter $\mu_{k+1} \in\left(0, \mu_{k}\right)$;

Set starting point for the next iteration to $x_{k+1}^{s}=x_{k}$; end (for)

Figure 3.5: Algorithm of the augmented Lagrangian method 
Based on the aforementioned illustration and discussion on the feasibility of the algorithms, we choose and implement the augmented Lagrangian method, which ensures the Non-twist constraints during the whole process of optimization. The detailed code segment can be found in Appendix.A. 


\section{Experimental Results}

In this chapter, we present several groups of experiments on $2 \mathrm{D}$ synthetic image data to demonstrate the improvement made by our proposed registration regularity. We have implemented the proposed Non-Twist regulariztion, and tested its effectiveness through two paired algorithms: the Demon's vs Demon's + Non-Twist and the DCT vs DCT + Non-Twist.

Since non-rigid image registration is an inherently ill-posed problem, where infinitely many solutions exist for a given input, there is no single best scheme to assess the performance of various registration algorithms. Numerous methods have been proposed in the literature attempting to establish a commonly accepted framework, but very few achieved the goal. An exception is the optical flow evaluation approach proposed by Barron et. al [5], with the support of an available public database. Vector angles between the ground truth and the estimated transformation vectors are employed to provide an indication how well a registration algorithm recovers the deformation field. In this chapter, we will utilize the same measure to demonstrate the improvements made by our Non-Twist algorithm, however, it should be noted that: being extremely faithful to the ground truth is not a prerequisite of a registration result being good, especially for complicated input cases as in our experiments. Other than making the two input image eventually look alike, our method has another goal, which is to maintain the regularity of the topology.

\subsection{Synthetic Sinusoidal Deformation}

In the experiments, the reference image (as in Figure 4.1.a) is a human brain MRI image, $256 \times 256$ demension and $1 \times 1 \mathrm{~mm}^{2}$ resolution, obtained from BrainWeb simulation [1]. For testing purposes, we can define a particular warp and apply it to an image, so that the deformation field can be known as the ground truth. Here, we use the sinusoidal displacement field [46] to deform the reference image: 


$$
\left\{\begin{array}{c}
x_{\text {new }}=\mathrm{x}_{\text {old }}+A_{x} \sin \left(\pi x_{\text {old }} / 32\right) \\
y_{\text {new }}=y_{\text {old }}+A_{y} \sin \left(\pi y_{\text {old }} / 32\right)
\end{array}\right.
$$

where $x_{\text {old }}$ and $y_{\text {old }}$ are coordinates of a pixel in the reference image 4.1.a, while $x_{\text {new }}$ and $y_{\text {new }}$ are coordinates of the corresponding point in the floating image 4.1.b. $A_{x}$ and $A_{y}$ are the limits of the maximum displacement distances along the $x$ and $y$ directions. The ground truth of the deformation field and the original differences between them are shown in Figure 4.1.c and d respectively.

\subsection{Comparative Results: Demon's vs Demon's + Non-Twist}

The Demon's algorithm uses Gaussian convolution to impose smoothness in the estimated deformation field, where the level of smoothness is controlled by the $\sigma$ of the Gaussian filter. Since the smoothness is evenly applied throughout the image domain, this penalty approach with a moderate Gaussian $\sigma$ does not prevent local twists. On the other hand, it is well-known that increasing $\sigma$ generally leads to an over-smoothing effect, which results in deteriorated precision in the registration results, as shown in Figure 4.2. 


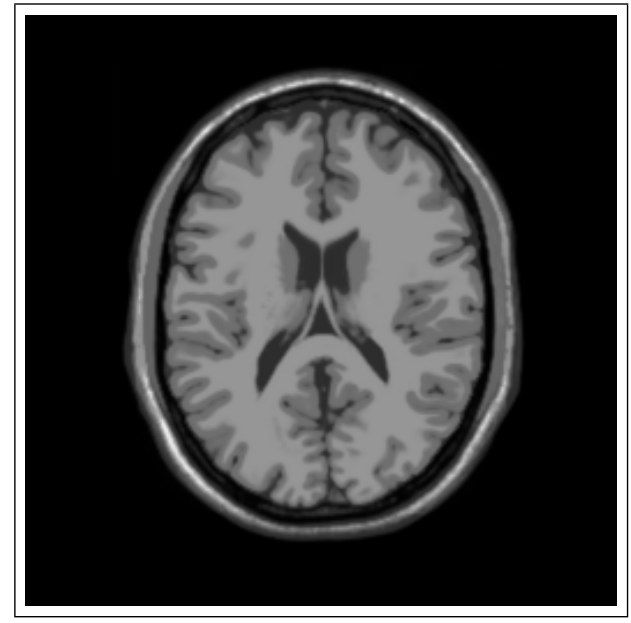

(a)

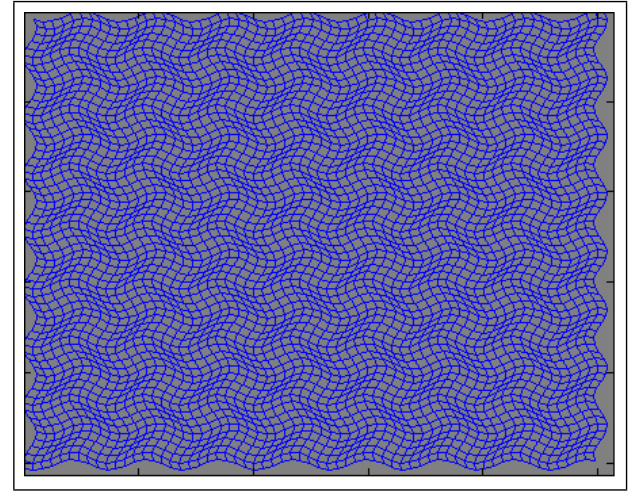

(c)

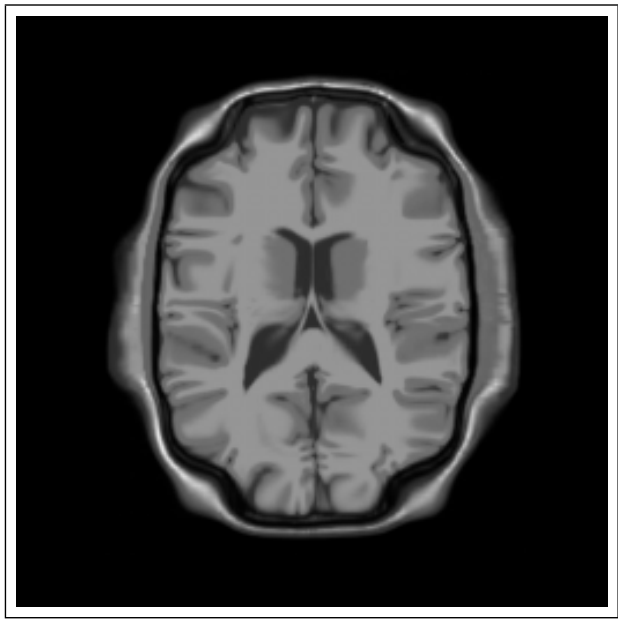

(b)

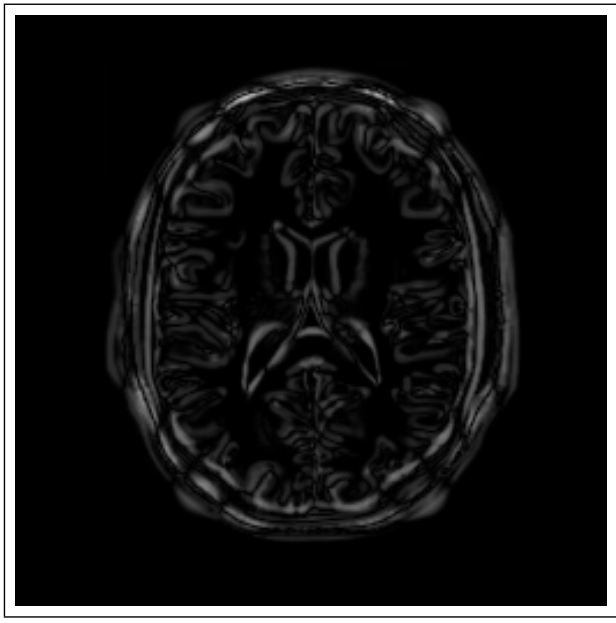

(d)

Figure 4.1: MR brain image. (a) Reference image (b) Floating image (c) ground truth of the deformation field (d) Original differences 


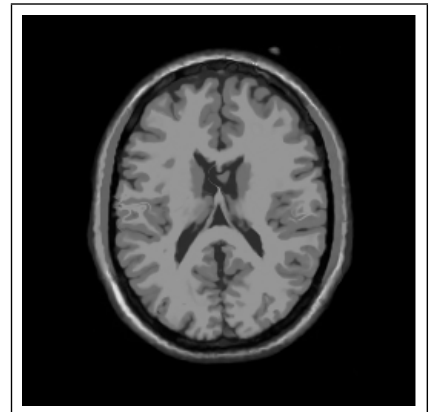

(a)

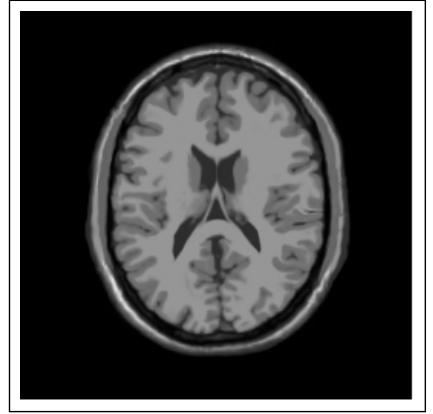

(b)

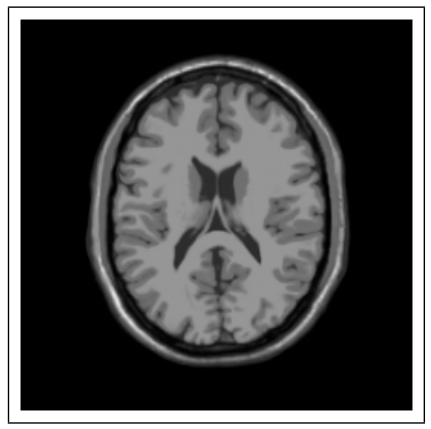

(c)

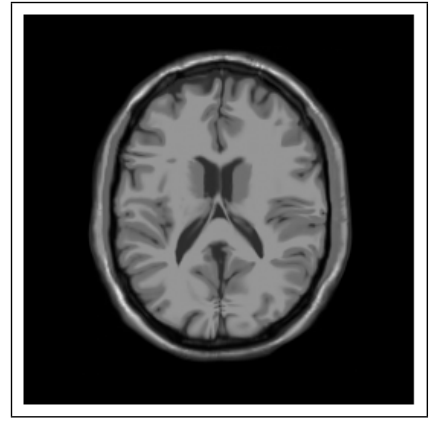

(d)

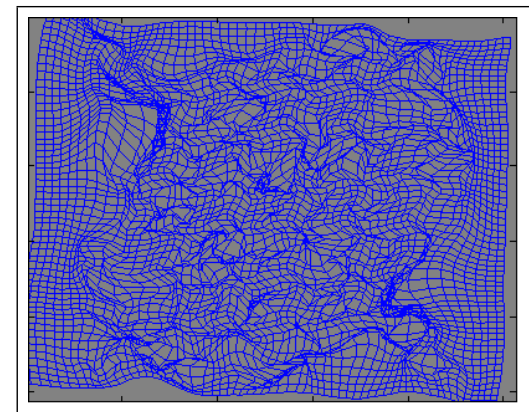

$\sigma=2$

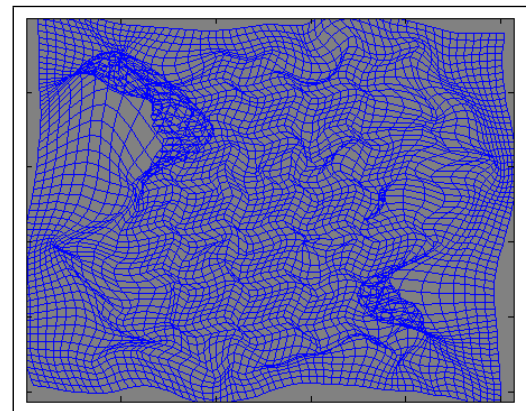

$\sigma=4$

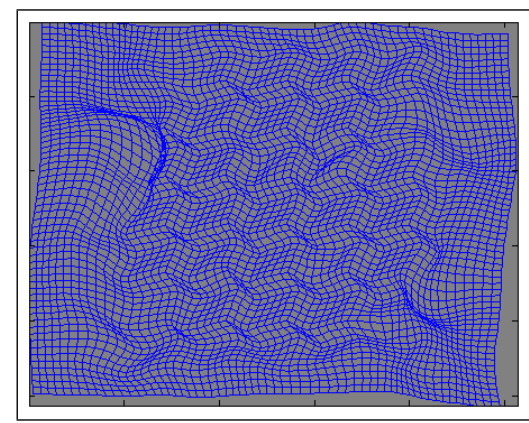

$\sigma=6$

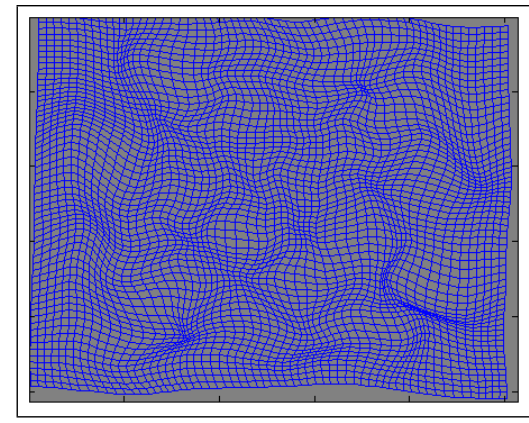

$\sigma=8$

Figure 4.2: Registration results by the Demon's alone. (The left figures are registered floating images, and the right figures are the corresponding transformation fields, with increasing smoothing- $\sigma$.) 
Figure 4.2 shows the registration results using the Demon's alone, with increasing smoothing power. From the results, we can directly observe: when $\sigma$ is small, which means the smoothing power is weak, the floating image is well registered towards the reference image. Nevertheless, the transformation field, which are full of obviously unreasonable twists, is deviated from the ground truth of the deformation field, as shown in Figure 4.1.c. When the smoothing power is increased, the twists in the transformation field are gone, as shown in Figure 4.2.d, but in the expense of deteriorating the registration effect.

Considering both the registration effect and the topology-preserved transformation field, we integrate our Non-Twist regularity into the Demon's method with $\sigma=6$. The registration results are shown in Figure 4.3.

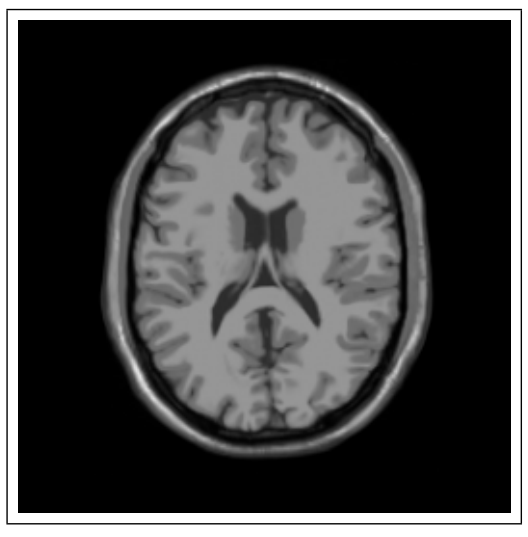

Demon's

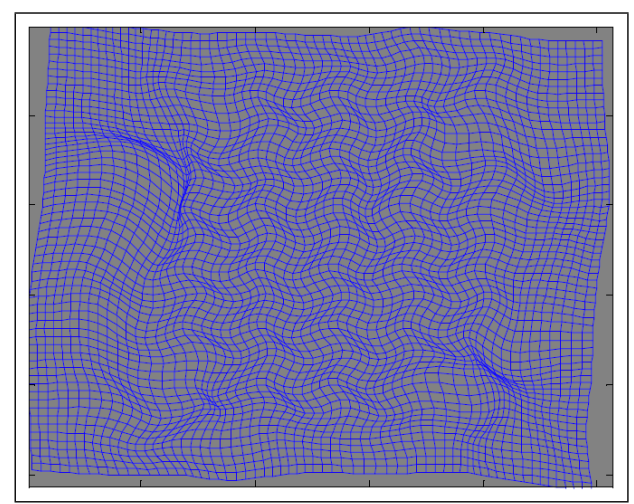

$\sigma=6$ with NonTwist Regularity

Figure 4.3: Registration results by the Demon's $(\sigma=6)$ with Non-Twist regularity.

Validation: As evident, local irregularities emerge in the upper left and bottom right brain boundary areas in Figure 4.2.c by using the Demon's alone, where no sufficient intensity detail exists to enforce the smoothness property. The estimated transformation field shown in Figure 4.3 comes from the new Demon's algorithm with our Non-Twist regularizer (concave version in this particular example). Obviously, all the irregularities 
have been driven away by the additional regularization component. Meanwhile, the registration effect is not affected negatively as in Figure 4.2.d.

Besides the direct observation, we also validate our results quantitatively. Since image registration is an ill-posed problem, where infinitely many solutions exist for a given input, there is no unique best approach to assess the performance of various registration algorithms. Nevertheless, we try to validate our proposed method on two purposes for medical image registration mentioned previously: 1) the decreased differences between input image pair, 2) and the legitimacy of the transformation field. Therefore, two aspects of properties are used to compare the results between the Demon's alone and Demon's + Non-Twist. Firstly, the sum of differences between the reference image and registrated image is used directly as the evidence for good matching, as shown in Table 4.1. Generally speaking, the smaller the sum of differences is, the better the registration performance is. Secondly, we try to quantify the accuracy for the deformation estimation. Here, the vector field of transformation, as shown in Figure 4.5, is used to extract the property: vector angles'differences [5]. For each pixel in vector field, a vector with certain angle and length exists to indicate its deformation. By comparing the angles with the ground truth, we can easily calculate the differences between them. statistical measures can be drawn from vector field, as shown in Table 4.2, to prove the effectiveness of topology-preservation.

Table 4.1: Sum of Differences: Demon's vs Demon's + Non-Twist

\begin{tabular}{ccc}
\hline \hline Group & Sum of Differences & Percentage of Improvement \\
\hline Original Differences & 612175 & \\
Demon's alone $(\sigma=6)$ & 95050 & $84.47 \%$ \\
Demon's with Non-Twist & 148607 & $75.72 \%$ \\
\hline
\end{tabular}




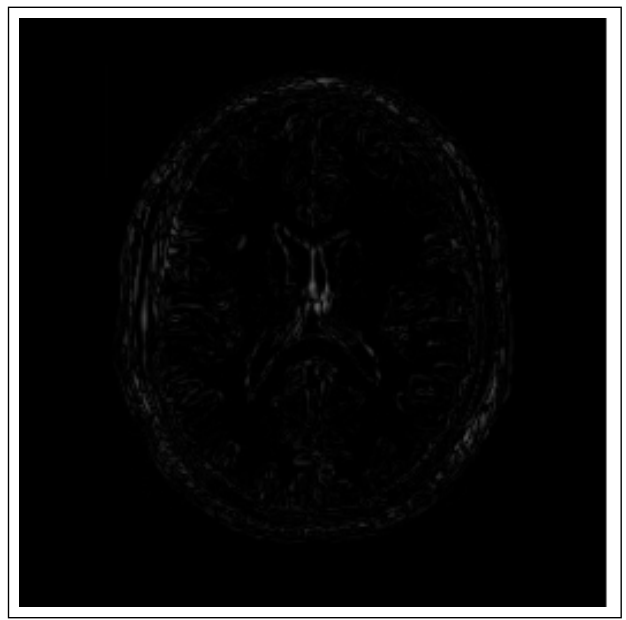

(a)Demon's alone

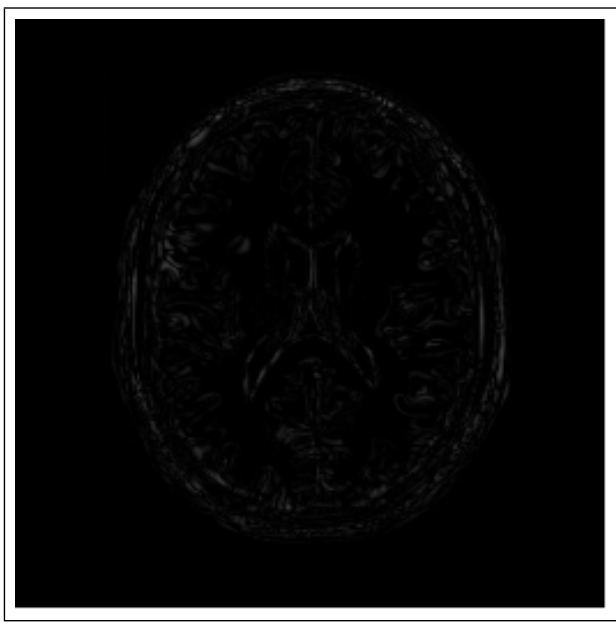

(b) Demon's with Non-Twist Regularity

Figure 4.4: Differences between the reference image and registered image.

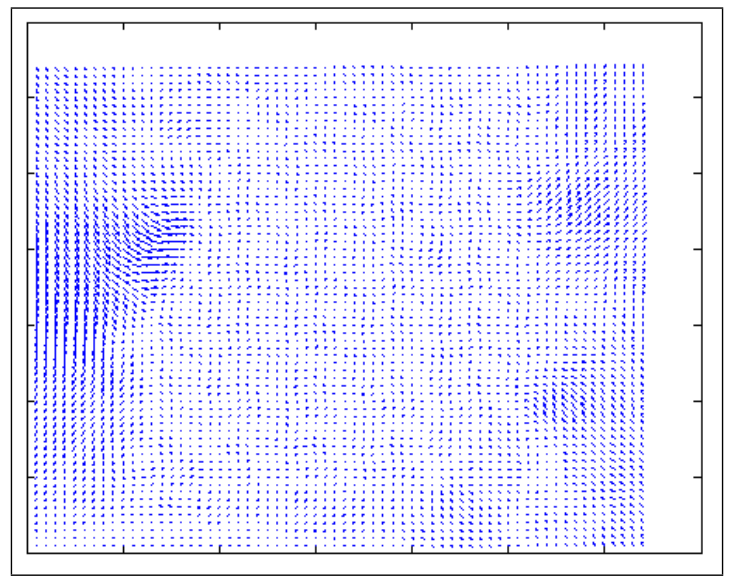

(a)Demon's alone

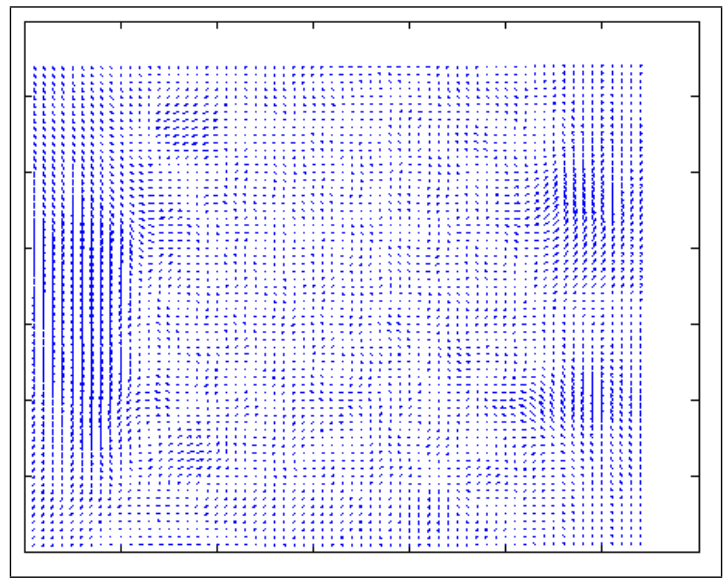

(b) Demon's with Non-Twist Regularity

Figure 4.5: Vector Field between the reference image and registrated image.

Based on Table 4.1 and 4.2, our Non-Twist regularization is not deteriorating the registration effect: the percentage of matching is as high as $75.72 \%$. Meanwhile, the mean of the vector angles' differences for the transformation field is $12.28^{\circ}$ deviated from the ground truth, which is much smaller than by using the Demon's method alone. 
Table 4.2: Average Angle Error: Demon's vs Demon's + Non-Twist

\begin{tabular}{ccc}
\hline \hline Group & Average Angle Error & Standard Deviation \\
\hline Demon's alone $(\sigma=6)$ & $18.11^{\circ}$ & 5.52 \\
Demon's with Non-Twist & $12.28^{\circ}$ & 5.01 \\
\hline
\end{tabular}

\subsection{Comparative Results: DCT vs DCT + Non-Twist}

The same input image pair has been used for the comparison of the DCT alone vs DCT + Non-Twist. Results are shown in Figure 4.6.a and Figure 4.6.b, respectively. The same number of coefficients, $32 \times 32$, are used in the experiments. It should be noted that the DCT alone has built-in global smoothness control stemming from its parametric deformation setup. However, the original method has no guaranteed mechanism to avoid local twists. The improvements with the added Non-Twist are obvious - Figure 2.1.b has much smoother grids and no visible local foldings. And also, the same quantitative comparisons are given by Tables 4.3 and 4.4 .

Table 4.3: Sum of Differences: DCT vs DCT + Non-Twist

\begin{tabular}{ccc}
\hline \hline Group & Sum of Differences & Percentage of Improvement \\
\hline Original Differences & 612175 & \\
DCT alone & 252973 & $58.68 \%$ \\
DCT with Non-Twist & 212532 & $65.29 \%$ \\
\hline
\end{tabular}

\subsection{Preliminary Design for GUI}

For the convenience of expected users, we also use the graphical user interface guide embedded in Matlab to build a preliminary GUI. The design for the main window, as shown 


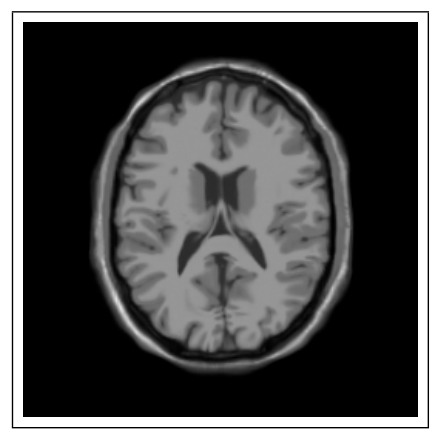

(a) DCT

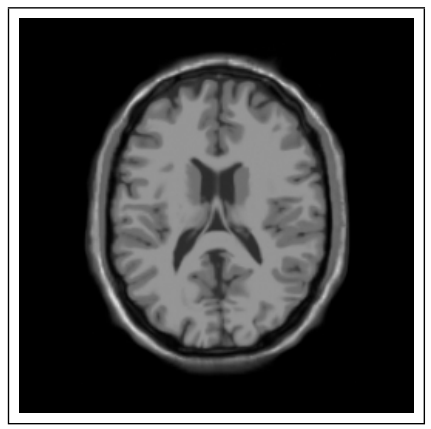

(b) DCT with Non-Twist

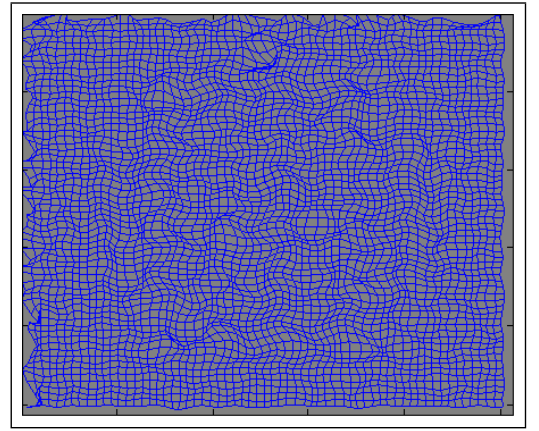

$32 \times 32$

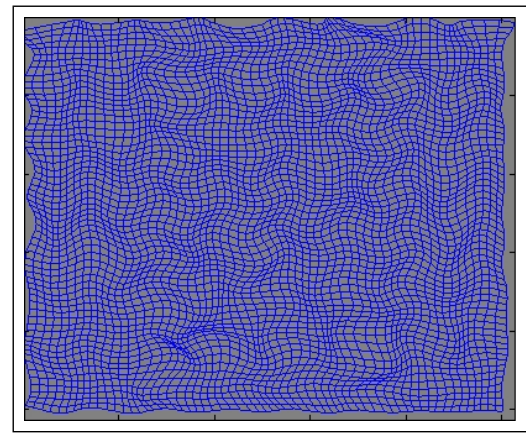

$32 \times 32$

Figure 4.6: Registration results by DCT alone and DCT with Non-Twist regularity. (The left figures are regitered floating images, and the right figures are the corresponding transformation fields. )

Table 4.4: Average Angle Error: DCT vs DCT + Non-Twist

\begin{tabular}{ccc}
\hline \hline Group & Average Angle Error & Standard Deviation \\
\hline DCT alone & $10.52^{\circ}$ & 4.68 \\
DCT with Non-Twist & $7.38^{\circ}$ & 3.90 \\
\hline
\end{tabular}

in Figure 4.9.a, integrates not only the functionalities that are mentioned in this thesis, e.g. the Demon's algorithm (as shown in Figure 4.9.b), the DCT algorithm, but also the related work by other members in our lab, which includes the point or curve registration, graph cut. Meanwhile, the design also maintains the potential for future deveplopment to be 


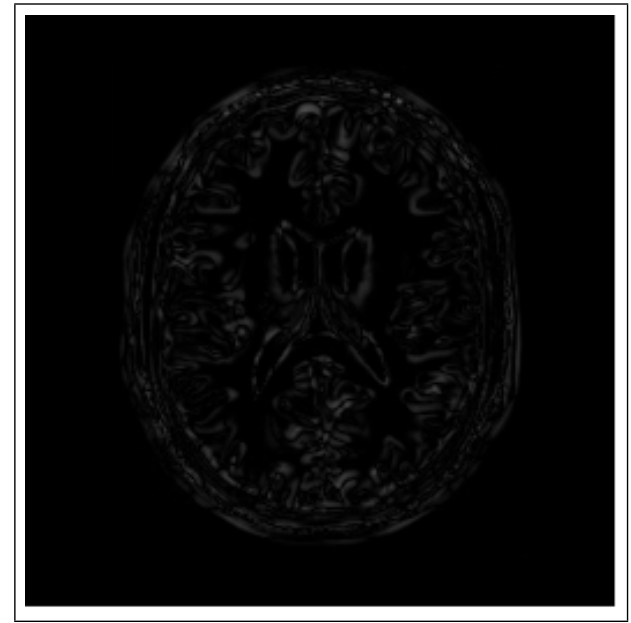

(a)DCT alone

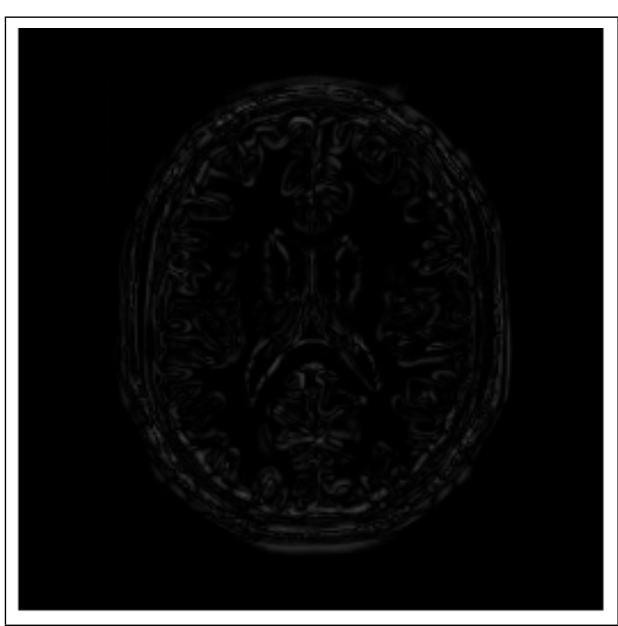

(b) DCT with Non-Twist Regularity

Figure 4.7: Differences between the reference image and registered image.

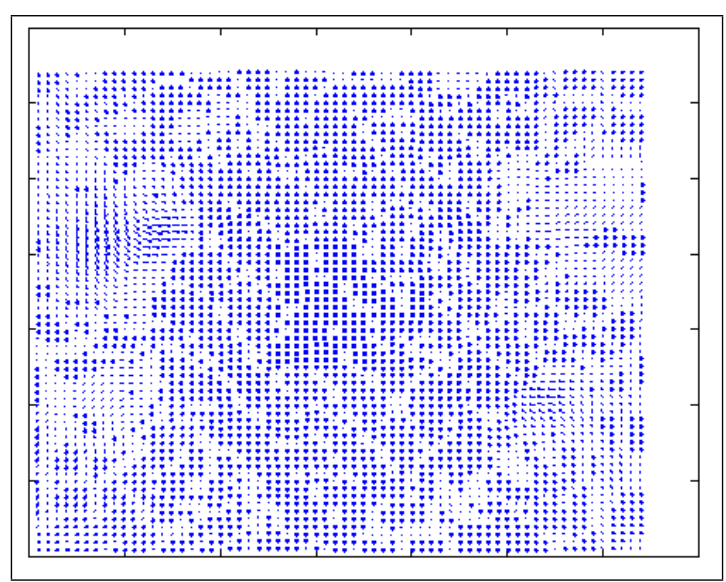

(a)DCT alone

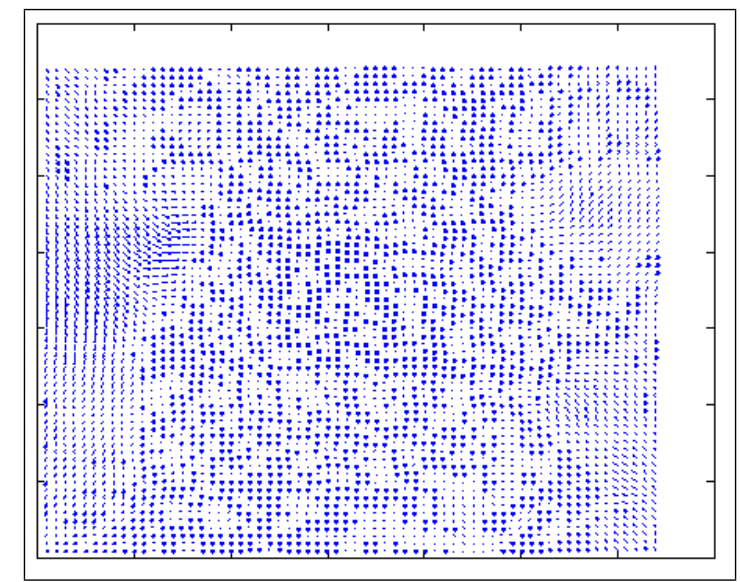

(b) DCT with Non-Twist Regularity

Figure 4.8: Vector Field between the reference image and registrated image.

a clinical registration package (as a SPM extension [23]) and disseminate it through the NITRC (Neuroimaging Informatics Tools and Resources Clearinghouse ) community. 


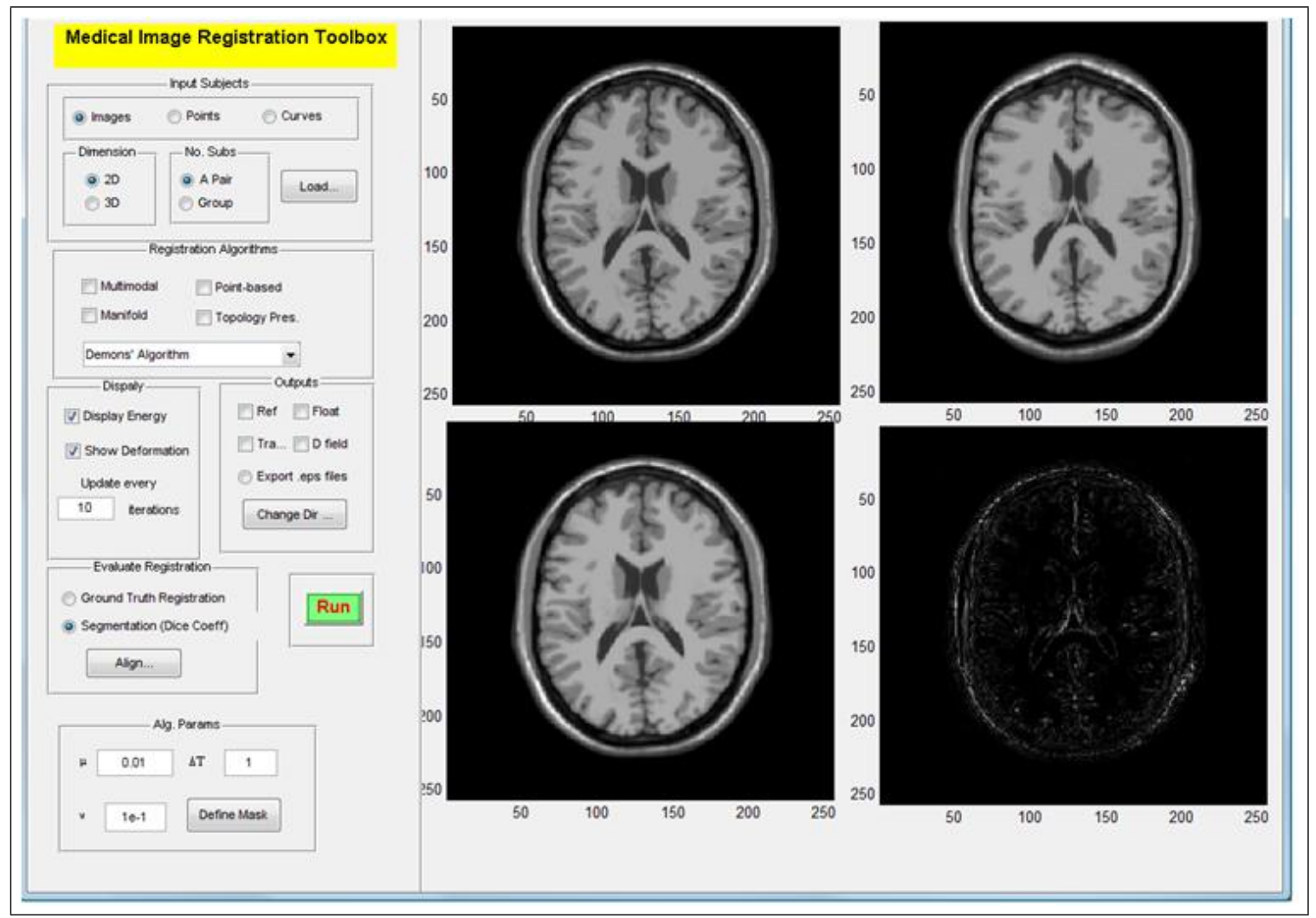

(a) Main interface

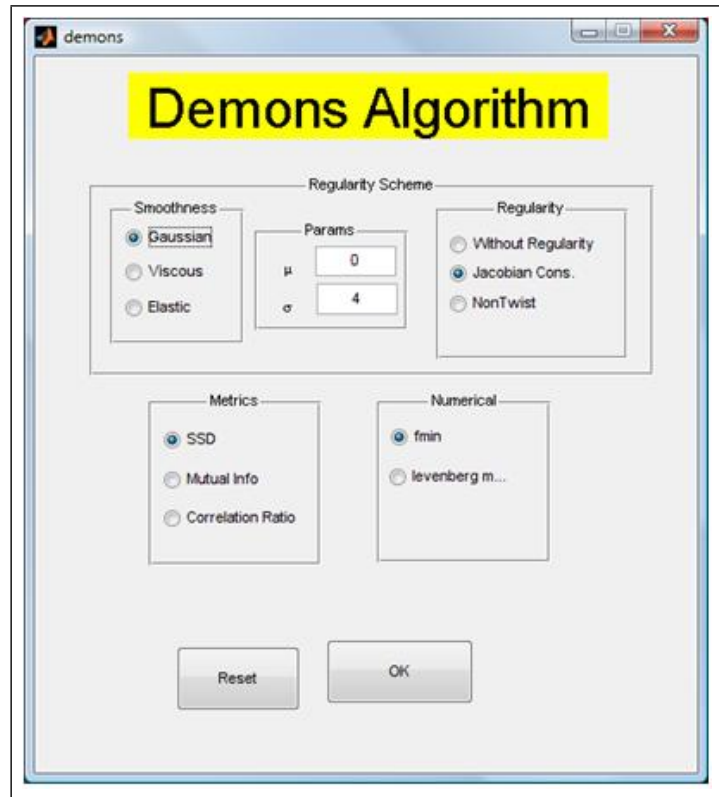

(b) Child window for Demon's algorithm

Figure 4.9: Medical Image Registration Toolbox. 


\section{Conclusion and Future Work}

Maintaining regularity and topology legitimacy is an important issue in medical image registration. Most of the previously published works are focused on using the Jacobians as the basis to specify regularity constraints, and most of the works only allow convex grid deformations. We identify the concave case and formulate the new NonTwist regularity under a straightforward point-line/surface orientation perspective. Our experimental results indicate that the added regularization can greatly improve the overall registration performance, especially in terms of smoothness and regularity.

A submission based on our proposed approach and the completed experiments has been accepted for publication in the conference of MIUA 2011. However, several aspects of the theory and implementation of our proposed work are still under development, which can be summarized as follows:

1. Conduct a more thorough quantitative assessment of the registration results.

Currently, the two quantitative measures are used to evaluate the registration results on both the differences minimization and the accuracy of the transformation estimation. The accuracy for the estimation would be potentially improved by removing the gloab rigid part. Besides, the image segmentation methods could also be used to extract the contours of brains, that can be used as a quantitative assessment.

2. Identify an application/presentation to demonstrate the difference between Convex and Concave constraints.

At present, we have theoretically differentiated the differences between the Convex and Concave gird updates, and also fulfilled the implementations of them. However, due to the fact that the pixel grid is in a tiny definition, as well as the interpolation involved in the update, the immediate and straightforward differences in a large scale can hardly be perceived by human vision. For this reason, a unique approach 
for presentation has to be designed, so that our proposed regularization can be demonstrated in both theoretical and experimental aspects.

3. Explore efficient approximation solutions (e.g. Generalized Regularization Networks based on Green function) to develop fast implementations. Also, develop a clinical registration package (as a SPM extension), and disseminate it through the NITRC (Neuroimaging Informatics Tools and Resources Clearinghouse ) community. 


\section{REFERENCES}

[1] BrainWeb:http://www.bic.mni.mcgill.ca/brainweb/.

[2] KKT:http://en.wikipedia.org/wiki/KarushTuckerconditions.

[3] J. Ashburner and K.J. Friston. Nonlinear spatial normalization using basis functions. Human brain mapping, 7(4):254-266, 1999.

[4] R. Bajcsy, R. Lieberson, and M. Reivich. A computerized system for the elastic matching of deformed radiographic images to idealized atlas images. Journal of computer assisted tomography, 7(4):618, 1983.

[5] J.L. Barron, DJ Fleet, and SS Beauchemin. Performance of optical flow techniques. International journal of computer vision, 12(1):43-77, 1994.

[6] F.L. Bookstein. Principal warps: Thin-plate splines and the decomposition of deformations. Pattern Analysis and Machine Intelligence, IEEE Transactions on, 11(6):567-585, 1989.

[7] D.E. Bredesen, R.V. Rao, and P. Mehlen. Cell death in the nervous system. Nature, 443(7113):796-802, 2006.

[8] C. Broit. Optimal registration of deformed images. 1981.

[9] Lisa Gottesfeld Brown. A survey of image registration techniques. ACM Computing Surveys, 24:325-376, 1992.

[10] MD Buhmann. Radial basis functions. Acta numerica, 9(-1):1-38, 2000.

[11] A. Burton. Thinking in perspective: critical essays in the study of thought processes. Routledge, 1978.

[12] R.H. Byrd, J.C. Gilbert, and J. Nocedal. A trust region method based on interior point techniques for nonlinear programming. Mathematical Programming, 89(1):149-185, 2000.

[13] G.E. Christensen, S.C. Joshi, and M.I. Miller. Volumetric transformation of brain anatomy. Medical Imaging, IEEE Transactions on, 16(6):864-877, 1997.

[14] G.E. Christensen, R.D. Rabbitt, and M.I. Miller. 3d brain mapping using a deformable neuroanatomy. Physics in medicine and biology, 39:609, 1994.

[15] G.E. Christensen, R.D. Rabbitt, and M.I. Miller. Deformable templates using large deformation kinematics. Image Processing, IEEE Transactions on, 5(10):1435-1447, 1996. 
[16] MK Chung, KJ Worsley, T. Paus, C. Cherif, DL Collins, JN Giedd, JL Rapoport, and AC Evans. A unified statistical approach to deformation-based morphometry. NeuroImage, 14(3):595-606, 2001.

[17] Mark Holden Derek L G Hill, Philipp G Batchelor and David J Hawkes. Medical image registration. Physics in Medicine and Biology, 46:R1-R45, 2001.

[18] James S. Duncan and Nicholas Ayache. Medical image analysis: Progress over two decades and the challenges ahead. IEEE Trans. Pattern Anal. Mach. Intell., 22(1):85$106,2000$.

[19] H. Edelsbrunner and E.P. Mucke. Simulation of simplicity: a technique to cope with degenerate cases in geometric algorithms. ACM Transactions on Graphics (TOG), 9(1):66-104, 1990.

[20] K. Feng and C. Shih. Mathematical theory of elastic structures. Springer (Berlin and New York and Beijing), 1996.

[21] B. Fischer and J. Modersitzki. Ill-posed medicinełan introduction to image registration. Inverse Problems, 24:034008, 2008.

[22] Society for Neuroscience. Brain Facts: A Primer on the Brain and Nervous System. Society for Neuroscience, 2008.

[23] K.J. Friston, J.T. Ashburner, S.J. Kiebel, T.E. Nichols, and W.D. Penny. Statistical parametric mapping: the analysis of funtional brain images. Elsevier/Academic Press, 2007.

[24] J.C. Gee, D.R. Haynor, M. Reivich, R. Bajcsy, et al. Finite element approach to warping of brain images. Citeseer, 1994.

[25] W.E.L. Grimson. A computational theory of visual surface interpolation. Philosophical Transactions of the Royal Society of London. B, Biological Sciences, 298(1092):395, 1982.

[26] E. Haber and J. Modersitzki. Image registration with guaranteed displacement regularity. International Journal of Computer Vision, 71(3):361-372, 2007.

[27] B. Karaçali and C. Davatzikos. Topology preservation and regularity in estimated deformation fields. In Information Processing in Medical Imaging, pages 426-437. Springer, 2003.

[28] W. Karush. Minima of functions of several variables with inequalities as side constraints. Master's thesis, Dept. of Mathematics, Univ. of Chicago, 1939. 
[29] H.W. Kuhn and A.W. Tucker. Nonlinear programming. In Proceedings of the Second Berkeley Symposium on Mathematical Statistics and Probability. July 31-August 12, 1950. Statistical Laboratory of the University of California, Berkeley. Berkeley, Calif.: University of California Press, 1951. 666 pp. Editor: Jerzy Neyman, p. 481-492, volume 1, pages 481-492, 1951.

[30] J. Kybic and M. Unser. Fast parametric elastic image registration. Image Processing, IEEE Transactions on, 12(11):1427-1442, 2003.

[31] J. B. Antoine Maintz and Max A. Viergever. A survey of medical image registration. Medical Image Analysis, 2:1-36, 1998.

[32] E. Meijering. A chronology of interpolation: From ancient astronomy to modern signal and image processing. Proceedings of the IEEE, 90(3):319-342, 2002.

[33] J. Modersitzki. Numerical methods for image registration. Oxford University Press, USA, 2004.

[34] O. Musse, F. Heitz, and J.P. Armspach. Topology preserving deformable image matching using constrained hierarchical parametric models. Image Processing, IEEE Transactions on, 10(7):1081-1093, 2001.

[35] V. Noblet, C. Heinrich, F. Heitz, and J.P. Armspach. 3-d deformable image registration: a topology preservation scheme based on hierarchical deformation models and interval analysis optimization. Image Processing, IEEE Transactions on, 14(5):553-566, 2005.

[36] J. Nocedal and S.J. Wright. Numerical optimization. Springer verlag, 1999.

[37] R.A. Novelline and L.F. Squire. Squire's fundamentals of radiology. Harvard Univ Pr, 2004.

[38] M.J.D. Powell. Approximation theory and methods. Cambridge Univ Pr, 1981.

[39] T. Rohlfing, C.R. Maurer Jr, D.A. Bluemke, and M.A. Jacobs. Volume-preserving nonrigid registration of $\mathrm{mr}$ breast images using free-form deformation with an incompressibility constraint. Medical Imaging, IEEE Transactions on, 22(6):730$741,2003$.

[40] D. Rueckert, L.I. Sonoda, C. Hayes, D.L.G. Hill, M.O. Leach, and D.J. Hawkes. Nonrigid registration using free-form deformations: application to breast $\mathrm{mr}$ images. Medical Imaging, IEEE Transactions on, 18(8):712-721, 1999.

[41] I.J. Schoenberg. Contributions to the problem of approximation of equidistant data by analytic functions. Quart. Appl. Math, 4(part A):45-99, 1946.

[42] L.L. Schumaker. Spline functions: basic theory. Cambridge Univ Pr, 2007. 
[43] M. Sdika. A fast nonrigid image registration with constraints on the jacobian using large scale constrained optimization. Medical Imaging, IEEE Transactions on, 27(2):271-281, 2008.

[44] I.M. Smith and D.V. Griffiths. Programming the finite element method. John Wiley \& Sons Inc, 2004.

[45] J.P. Thirion. Image matching as a diffusion process: an analogy with maxwell's demons. Medical image analysis, 2(3):243-260, 1998.

[46] Y. Wang and L. Staib. Elastic model based non-rigid registration incorporating statistical shape information. Medical Image Computing and Computer-Assisted InterventationtMICCAI98, pages 1162-1173, 1998.

[47] D.H. Warren and E.R. Strelow. Electronic spatial sensing for the blind. Nijhoff, 1985.

[48] Barbara Zitovф and Jan Flusser. Image registration methods: a survey. Image and Vision Computing, 21:977-1000, 2003. 


\section{Appendix: Matlab Code}

\section{A.1 Function for Augmented Lagrangian method}

function $[\mathrm{x}]=\mathrm{ALM}$ (funfcn, $\mathrm{x} \_$init, $\mathrm{CEQ}$ )

$\%$ input

\% funfcn: SSD objective function

$\%$ x: $\quad$ starting points

\%\% CEQ: nonlinear equality constraint

$\%$ initialize

$\%$ construct the ALM objective functive

$$
\begin{aligned}
& \text { miu }=1 ; \\
& \text { lamda = 1; } \\
& \text { x=x_init; } \\
& \text { tol1= 1e-5; } \\
& \text { tol2= } 1 \mathrm{e}-8 ;
\end{aligned}
$$

for $k=0: 1000$

$\%$ gradL = gradALMobjective (funfcn, CEQ , $\mathrm{x}$, lamda, miu);

$[\mathrm{C}, \mathrm{h}]=\mathrm{CEQ}(\mathrm{x})$;

Obj= $\quad=\operatorname{funfcn}(\mathrm{x})$; 


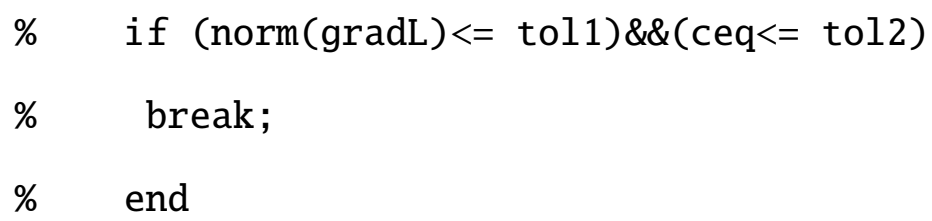

\% Choose between two minimization methods: fminserch and fminlbfgs

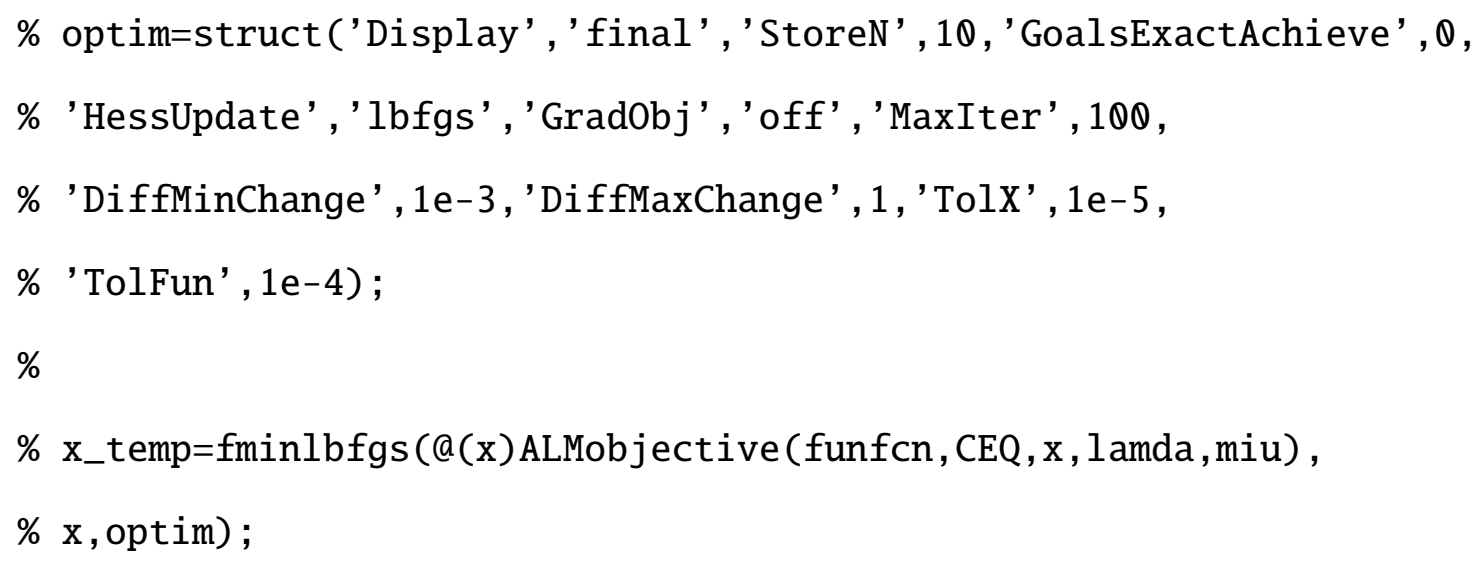




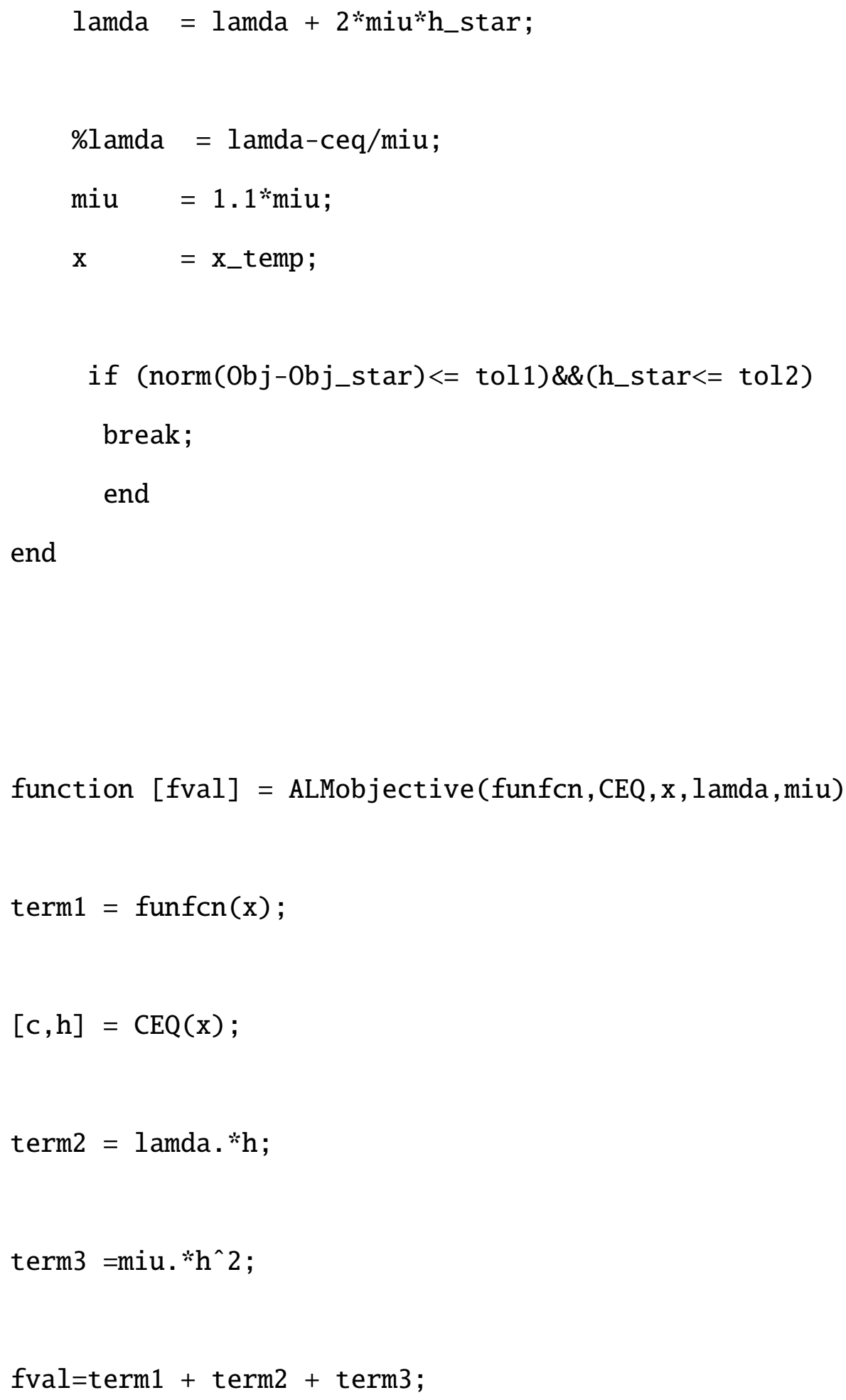


function $[\mathrm{grad}]=$ gradALMobjective $($ funfcn, $\mathrm{CEQ}, \mathrm{x}$, lamda, $\mathrm{miu})$

DiffMaxChange $=10$;

DiffMinChange $=1$;

\% Calculate gradient with forward difference

$\%$ if not provided by the function

$\operatorname{grad}=\operatorname{zeros}($ length $(\mathrm{x}), 1)$;

\%fval=dct_registration_SSD ( $x$, Imoving, Istatic , D1 , D2 , M , N) ;

fval=ALMobjective (funfcn, CEQ , x , lamda , miu);

gstep $=1$

if(gstep $>$ DiffMaxChange), gstep $=1$; end

if(gstep $<$ DiffMinChange), gstep $=1$; end

for $i=1$ : length $(x)$,

$x_{-}$temp $=x ; x_{-} \operatorname{temp}(i)=x_{-}$temp $(i)+g s t e p ;$

x_temp';

\%fval_g=dct_registration_SSD (x_temp, Imoving, Istatic, D1, D2 , M , N) ; fval_g=ALMobjective (funfcn, CEQ, x_temp, lamda , miu) ;

\% data.timeExtern=data.timeExtern+toc (timem);

$\operatorname{grad}(i)=\left(f v a l \_g-f v a l\right) / g s t e p ;$

end 


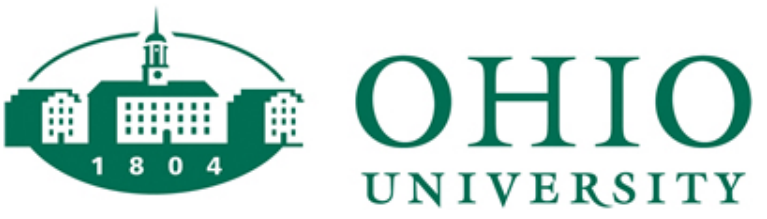

Thesis and Dissertation Services 